\title{
FRICAT: A FIRST catalog of FR I radio galaxies
}

\author{
A. Capetti ${ }^{1}$, F. Massaro ${ }^{2}$, and R. D. Baldi ${ }^{3}$ \\ 1 INAF-Osservatorio Astrofisico di Torino, via Osservatorio 20, 10025 Pino Torinese, Italy \\ e-mail: capetti@oato.inaf.it \\ 2 Dipartimento di Fisica, Università degli Studi di Torino, via Pietro Giuria 1, 10125 Torino, Italy \\ 3 Department of Physics and Astronomy, University of Southampton, Highfield, SO17 1BJ, UK
}

Received 11 July 2016 / Accepted 30 September 2016

\begin{abstract}
We built a catalog of 219 FR I radio galaxies (FR Is), called FRICAT, selected from a published sample and obtained by combining observations from the NVSS, FIRST, and SDSS surveys. We included in the catalog the sources with an edge-darkened radio morphology, redshift $\leq 0.15$, and extending (at the sensitivity of the FIRST images) to a radius $r$ larger than $30 \mathrm{kpc}$ from the center of the host. We also selected an additional sample (sFRICAT) of 14 smaller $(10<r<30 \mathrm{kpc})$ FR Is, limiting to $z<0.05$. The hosts of the FRICAT sources are all luminous $\left(-21 \gtrsim M_{r} \gtrsim-24\right)$, red early-type galaxies with black hole masses in the range $10^{8} \lesssim M_{\mathrm{BH}} \lesssim 3 \times 10^{9} M_{\odot}$; the spectroscopic classification based on the optical emission line ratios indicates that they are all low excitation galaxies. Sources in the FRICAT are then indistinguishable from the FR Is belonging to the Third Cambridge Catalogue of Radio Sources (3C) on the basis of their optical properties. Conversely, while the 3C-FR Is show a strong positive trend between radio and [O III] emission line luminosity, these two quantities are unrelated in the FRICAT sources; at a given line luminosity, they show radio luminosities spanning about two orders of magnitude and extending to much lower ratios between radio and line power than 3C-FR Is. Our main conclusion is that the 3C-FR Is just represent the tip of the iceberg of a much larger and diverse population of FR Is.
\end{abstract}

Key words. galaxies: active - galaxies: jets

\section{Introduction}

Fanaroff \& Riley (1974) introduced the first classification scheme for extragalactic radio sources with large-scale structures (i.e., greater than $\sim 15-20 \mathrm{kpc}$ in size). They proposed to distinguish radio sources into two main classes on the basis of the relation between relative positions of regions of high and low surface brightness in their extended components. This scheme was based on the ratio $R_{\mathrm{FR}}$ of the distance between the regions of highest surface brightness on opposite sides of the central host galaxy to the total extent of the source up to the lowest brightness contour in the radio images. Radio sources with $R_{\mathrm{FR}}<0.5$ were placed in Class I (i.e., the edge-darkened FR Is) and sources with $R_{\mathrm{FR}}>0.5$ in Class II (i.e., the edge-brightened FR IIs).

This morphology-based classification scheme was also linked to their intrinsic power, when Fanaroff and Riley found that all sources in their sample with luminosity at $178 \mathrm{MHz}$ smaller than $2 \times 10^{25} \mathrm{~W} \mathrm{~Hz}^{-1} \mathrm{sr}^{-1}$ (for a Hubble constant of $50 \mathrm{~km} \mathrm{~s}^{-1} \mathrm{Mpc}^{-1}$ ) were classified as FR I while the brighter sources all were FR II. The luminosity distinction between FR classes is fairly sharp at $178 \mathrm{MHz}$ but their separation is cleaner in the optical-radio luminosity plane, implying that the FR I/FR II dichotomy depends on optical and radio luminosity (Ledlow \& Owen 1996).

The selection of large and well-defined samples of radio galaxies is of great importance to properly address several issues, such as building their luminosity functions, exploring the properties of their hosts, studying their environment and their cosmic evolution, and comparing the results obtained for the different classes of radio-galaxies for radio-quiet active nuclei and for the population of quiescent galaxies.

In particular, the number of known FR I radio galaxies is rather small. For example the Third Cambridge Catalogue of Radio Sources (3C; Bennett 1962) includes less than 30 FR Is. The second Bologna sample (B2; Colla et al. 1975; Fanti et al. 1978 ) is formed by $\sim 100$ radio galaxies of lower luminosity than those of the $3 \mathrm{C}$; most of these have a luminosity below the FR I/FR II transition and about half of them of are FR I. These samples are not sufficiently large to address the issues listed above properly. Furthermore, as these samples are selected with a rather high flux threshold, they present a limited (and possibly statistically biased) view of the FR I population.

The advent of large area surveys opens the opportunity to set the results on several key issues on strong statistical foundations. In particular, the radio, infrared, and optical observations available thanks to recent large-area surveys are a unique tool in the analysis of the radio galaxies and quasars, since they allow us to identify large numbers of radio sources, obtain spectroscopic redshifts, and determine the properties of their hosts. Best et al. (2005), Baldi \& Capetti (2010), and Best \& Heckman (2012) already used the extensive multifrequency information available to analyze the properties of the population of low redshift radio emitting AGN. We here also consider the radio morphological information and explore the possibility to create the first catalog of FR I radio galaxies selected on the basis of radio and optical data, which we call the FRICAT.

This paper is organized as follows. In Sect. 2 we present the selection criteria of the sample of FR Is, whose completeness 
is discussed in Sect. 3. The radio and optical properties of the selected sources are presented in Sect. 4 and discussed in Sect. 5. Section 6 is devoted to our summary and conclusions.

Throughout the paper we adopt a cosmology with $H_{0}=$ $67.8 \mathrm{~km} \mathrm{~s}^{-1} \mathrm{Mpc}^{-1}, \Omega_{\mathrm{M}}=0.308$, and $\Omega_{\Lambda}=0.692$ (Planck Collaboration XIII 2016).

For our numerical results, we use c.g.s. units unless stated otherwise. Spectral indices $\alpha$ are defined by the usual convention on the flux density, $S_{v} \propto v^{-\alpha}$. The SDSS magnitudes are in the $\mathrm{AB}$ system and are corrected for the Galactic extinction; WISE magnitudes are instead in the Vega system and are not corrected for extinction since, as shown by, for example, D'Abrusco et al. (2014), such correction affects only the magnitude at $3.4 \mu$ of sources lying at low Galactic latitudes (and by less than $\sim 3 \%$ ).

\section{Sample selection}

We searched for FR I radio galaxies in the sample of 18286 radio sources built by Best \& Heckman (Best \& Heckman 2012, hereafter the BH12 sample) by limiting our search to the subsample of objects in which, according to these authors, the radio emission is produced by an active nucleus. They cross-matched the optical spectroscopic catalogs produced by the group from the Max Planck Institute for Astrophysics and The Johns Hopkins University (Brinchmann et al. 2004; Tremonti et al. 2004) based on data from the data release 7 of the Sloan Digital Sky Survey (DR7/SDSS; Abazajian et al. 2009) ${ }^{1}$, with the National Radio Astronomy Observatory Very Large Array Sky Survey (NVSS; Condon et al. 1998) and the Faint Images of the Radio Sky at Twenty centimeters survey (FIRST; Becker et al. 1995) adopting a radio flux density limit of $5 \mathrm{mJy}$ in the NVSS. We focused on the 3,357 sources with redshift $z<0.15$.

We visually inspected all the FIRST images of each individual source and preserved only those whose radio emission reaches a distance of at least $30 \mathrm{kpc}$ from the center of the optical host at the sensitivity of the FIRST images. Such a radius corresponds to $11^{\prime \prime} .4$ for the farthest objects; this ensures that all the 741 selected sources are well resolved with the $5^{\prime \prime}$ resolution of the FIRST images. This permitted us to properly explore their morphology. The reference surface brightness level adopted is $0.45 \mathrm{mJy} /$ beam (approximatively three times the typical $\mathrm{rms}$ of the FIRST images) for the objects at $z=0.15$. The brightness level is increased by a factor $[(1+0.15) /(1+z)]^{4}$ for closer objects to compensate for the cosmological surface brightness dimming; this level corresponds to a correction factor of $\sim 1.75$ for $z=0$. We also applied a $k$ correction by assuming a spectral index of 0.7 , which is typical of the extended radio emission; in this case the correction is rather small, amounting to at most $\sim 10 \%$.

We adopted a purely morphological classification based on the radio structure shown by the FIRST images. The original FR I definition corresponds to "a great diversity of structure" (Fanaroff \& Riley 1974), and it is not always of easy application. We adopted rather strict criteria for a positive classification for the selection of the FIRST sample of FR Is. We limited our selection to the sources showing one-sided or two-sided jets in which the surface brightness is generally decreasing along its whole length, lacking of any brightness enhancement at the jet end. We allowed for bent jets and we thus included narrow angle tail (NAT; Rudnick \& Owen 1977) sources; conversely, we excluded the sources in which a substantial brightening occurs along the jet, thus excluding, for example, wide angle tail (WAT; Owen \& Rudnick 1976) objects.

Available at http://www . mpa-garching.mpg.de/SDSS/.
The three authors performed this analysis independently and we included only the sources for which a FR I classification is proposed by at least two of us.

The resulting sample, to which we refer as FRICAT, is formed by 219 FR Is. In Fig. 1 we present the FIRST images of the first 12 FRICAT sources selected to illustrate the outcome of our selection. Images of all FRICAT objects are available in the Appendix. Their main properties are presented in Table B.1, where we report the SDSS name, redshift, and NVSS $1.4 \mathrm{GHz}$ flux density (from BH12). The [O III] line flux, the $r$-band SDSS AB magnitude, $m_{r}$, the Dn(4000) index (see Sect. 4 for the definition of the $\mathrm{Dn}(4000)$ ), and the stellar velocity dispersion $\sigma_{*}$ are instead from the MPA-JHU DR7 release of spectrum measurements. The concentration index $C_{r}$ was obtained for each source directly from the SDSS database. For sake of clarity, errors are not shown in the table; we estimated a median error of 0.08 on $C_{r}$, of 0.03 on $\mathrm{Dn}(4000)$, of 0.004 magnitudes on $m_{r}$, and of $9 \mathrm{~km} \mathrm{~s}^{-1}$ on $\sigma_{*}$. Finally we list the resulting radio and line luminosity, and the black hole masses estimated from the stellar velocity dispersion and the relation $\sigma_{*}-M_{\mathrm{BH}}$ of Tremaine et al. (2002). The error in the $M_{\mathrm{BH}}$ is dominated by the spread of the relation used (rather than by the errors in the measurements of $\sigma_{*}$ ) resulting in an uncertainty of a factor $\sim 2$.

The limited resolution of FIRST imposes a minimum size of $30 \mathrm{kpc}$ to the FR Is. We selected (with the same criteria discussed above) a second sample of FR Is extending to smaller radii, $10<r<30 \mathrm{kpc}$, to consider also less extended radio sources. We limited this sample to nearby objects $(z<0.05)$ to preserve a sufficient spatial resolution. The images of these 14 sources, forming the "small" FR Is sample (hereafter sFRICAT), are presented in Fig. 2 and their properties are listed in Table B.2.

\section{The completeness of the FRICAT samples}

We now discuss the completeness of the sample related to the radio and optical selection.

Concerning the radio selection, the BH12 includes sources with a NVSS flux density larger than $5 \mathrm{mJy}$. However, our selection also depends on the brightness distribution in the FIRST images. Therefore we might be missing objects characterized by, for example, diffuse emission not reaching the $3 \sigma$ limit in these higher resolution images; furthermore, some extended emission might be resolved out, and missed, by the FIRST maps.

In the left panel of Fig. 3, we show the distribution of the flux density at $1.4 \mathrm{GHz}$ (i.e., $F_{1.4}$ ) for all the sources belonging to the FRICAT; this flux density peaks at $\sim 50 \mathrm{mJy}$ and extends up to 5 Jy. The brightest source, FRICAT 1416+1048, is the only objects belonging to the $3 \mathrm{C}$ sample, 3C 296. Below the peak the sources density decreases and there are only two objects between 5 and $10 \mathrm{mJy}$. This flux distribution indicates that indeed the completeness limit of FRICAT is higher than the original $5 \mathrm{mJy}$ and can be set at $\sim 50 \mathrm{mJy}$.

As for the optical selection of the sample, according to Montero-Dorta \& Prada (2009), the redshift completeness of the SDSS decreases with decreasing apparent magnitude, starting from $\sim 90 \%$ at the SDSS spectroscopic limit of $r=17.77$ and reaching $\sim 50 \%$ at $r=11.75$. Most of the incompleteness is due to the SDSS fiber cladding, which prevents fibers on any given plate from being placed closer than 55" apart. For brighter (and more extended) objects other effects become important, such as the superposition of bright saturated stars on the target. 
A. Capetti et al.: FRICAT: A FIRST catalog of FR I radio galaxies
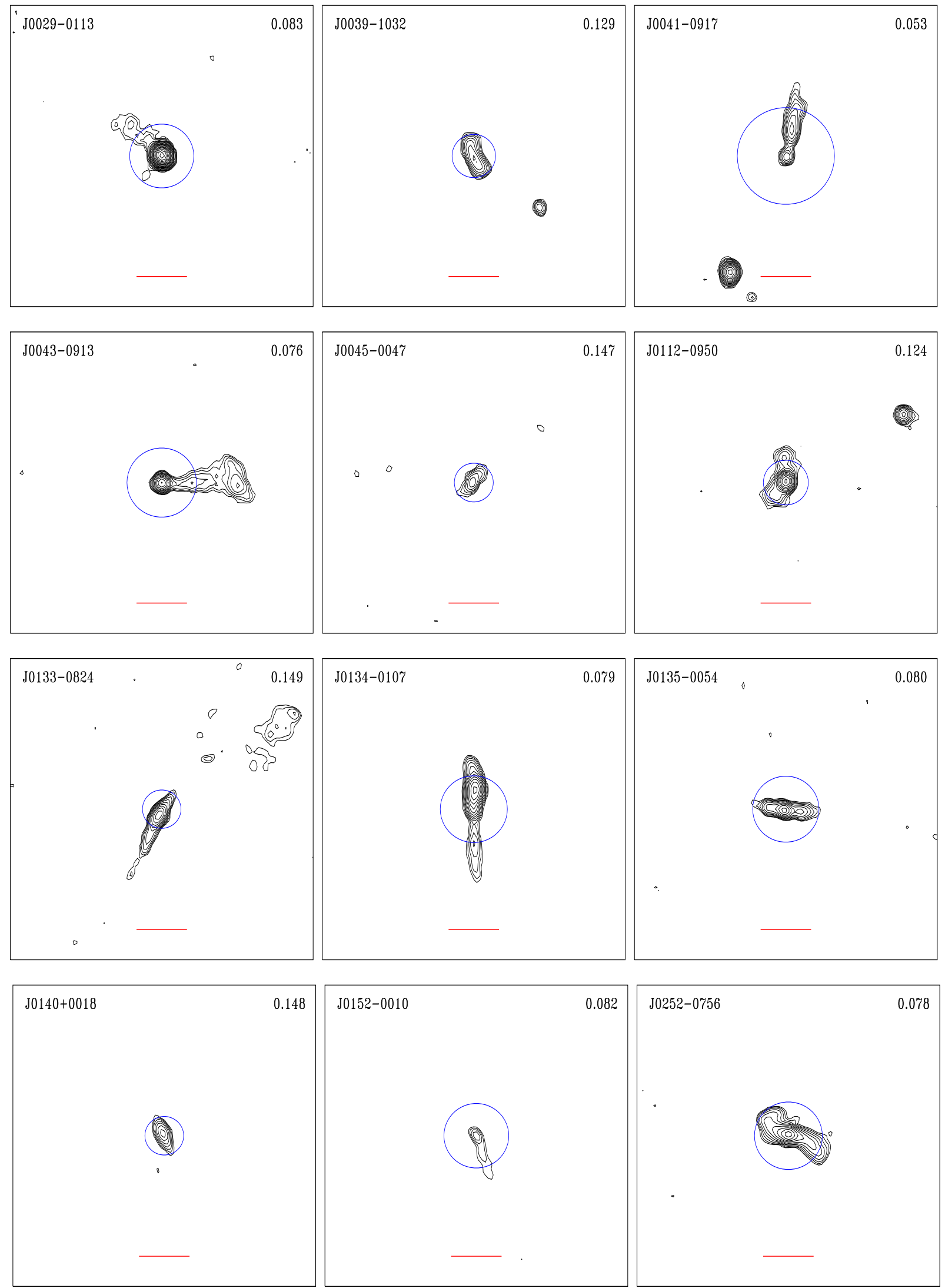

Fig. 1. FIRST images of the first 12 FRICAT sources. Contours, corrected for cosmological effects to a redshift of $z=0.15$, are drawn starting from $0.45 \mathrm{mJy} /$ beam and increase with a geometric progression with a common ratio of $\sqrt{2}$. The field of view is $3^{\prime} \times 3^{\prime}$; the red tick at the bottom is $30^{\prime \prime}$ long. The blue circle is centered on the host galaxy and has a radius of $30 \mathrm{kpc}$. The sources FRICAT name and redshift are reported in the upper corners. 

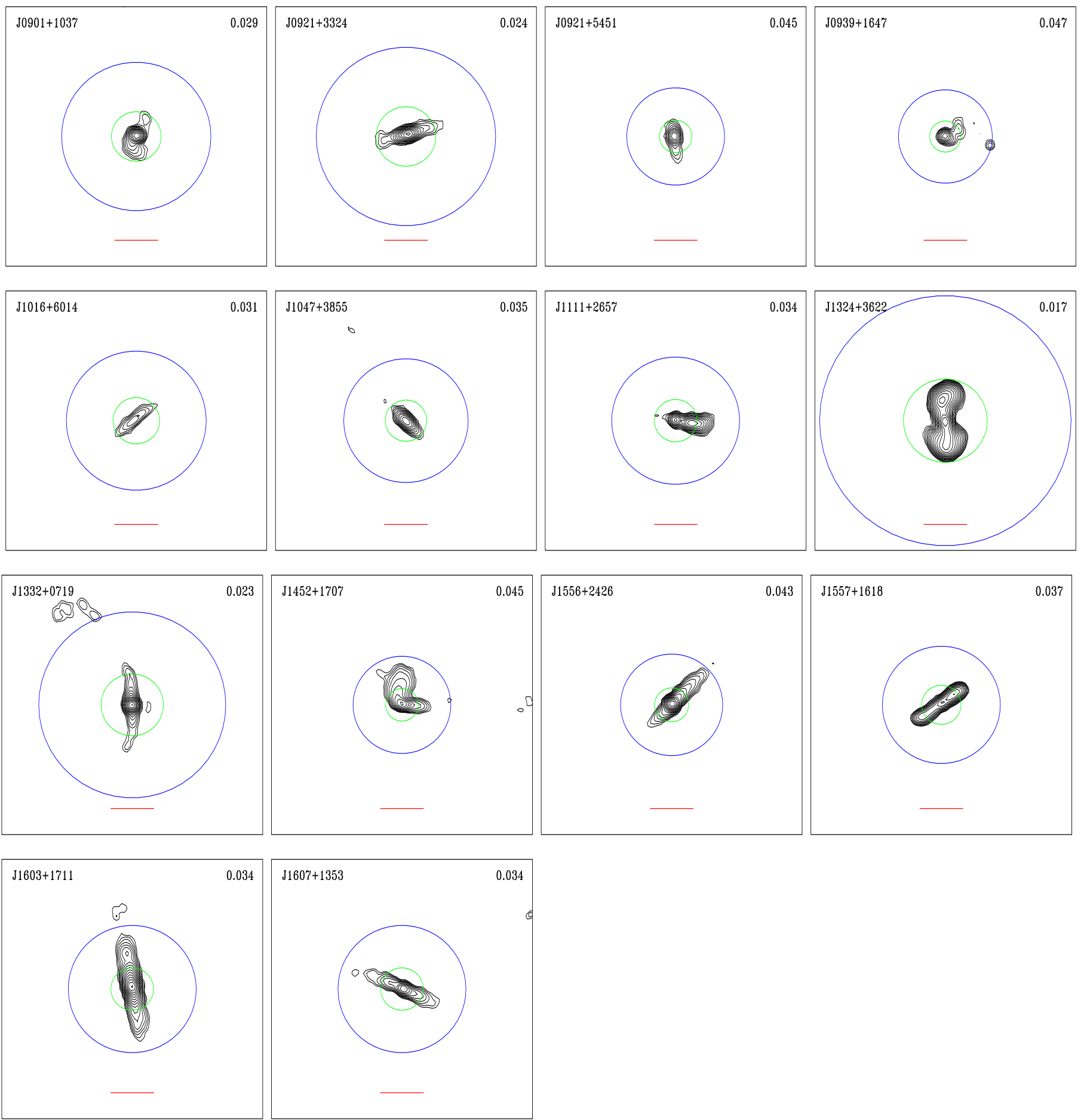

Fig. 2. FIRST images of the 14 sFRICAT sources selected at $z<0.05$ and extended between 10 and $30 \mathrm{kpc}$. The green (blue) circle is centered on the host galaxy and has a radius of $10(30) \mathrm{kpc}$. The sources sFRICAT name and redshift are reported in the upper corners.

In the right panel of Fig. 3, we show the distribution of the $r$ magnitude of the FRICAT hosts. The vast majority of them fall in the magnitude range of the SDSS main galaxies sample (Strauss et al. 2002; $17.77<r<14.5$ ); a bright tail of objects (also including most of the sFRICAT hosts) is present but it drops to zero well before the redshift completeness is significantly reduced.

Thus both FR Is catalogs (FRICAT and sFRICAT) are statistically complete at level of $\sim 90 \%$ in the optical energy range. However, it is worth mentioning that this extremely low level of incompleteness is only due to a random loss of $\sim 10 \%$ of the potential spectroscopic targets (see, e.g., Zehavi et al. 2002).

\section{FRICAT hosts and radio properties}

\subsection{Hosts properties}

All selected FR Is are classified as low excitation galaxies (LEG) by Best \& Heckman (2012) based on the ratios of the optical emission lines in their SDSS spectra. There are only four exceptions and these are sources that cannot be classified 

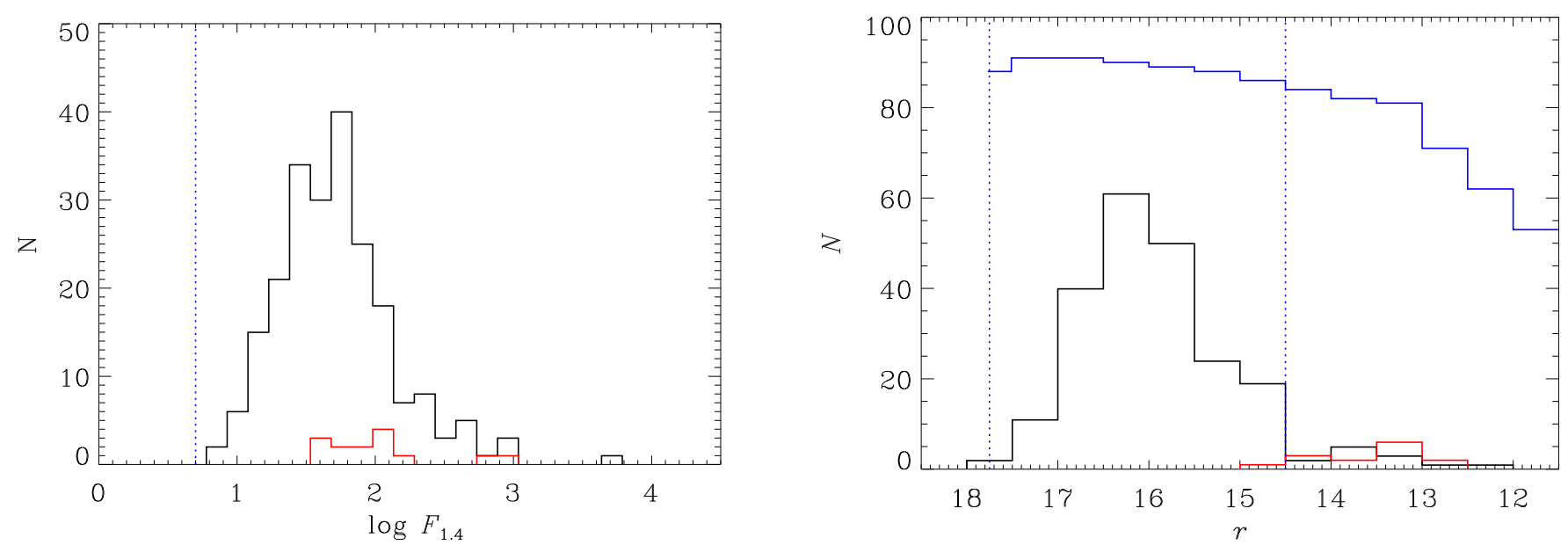

Fig. 3. Left: distribution of the NVSS fluxes of the 219 FRICAT sources; the red histogram is for the 14 sFRICAT sources. The vertical blue dotted line indicates the 5 mJy limits of the BH12 sample. Right: the black curve shows the $r$-band magnitude distribution of the FRICAT hosts (the red histogram is for the sFRICAT sources); the vertical dotted lines indicate the limits defining the SDSS main galaxies sample. The blue histogram report the SDSS completeness in percentage from Montero-Dorta \& Prada (2009).
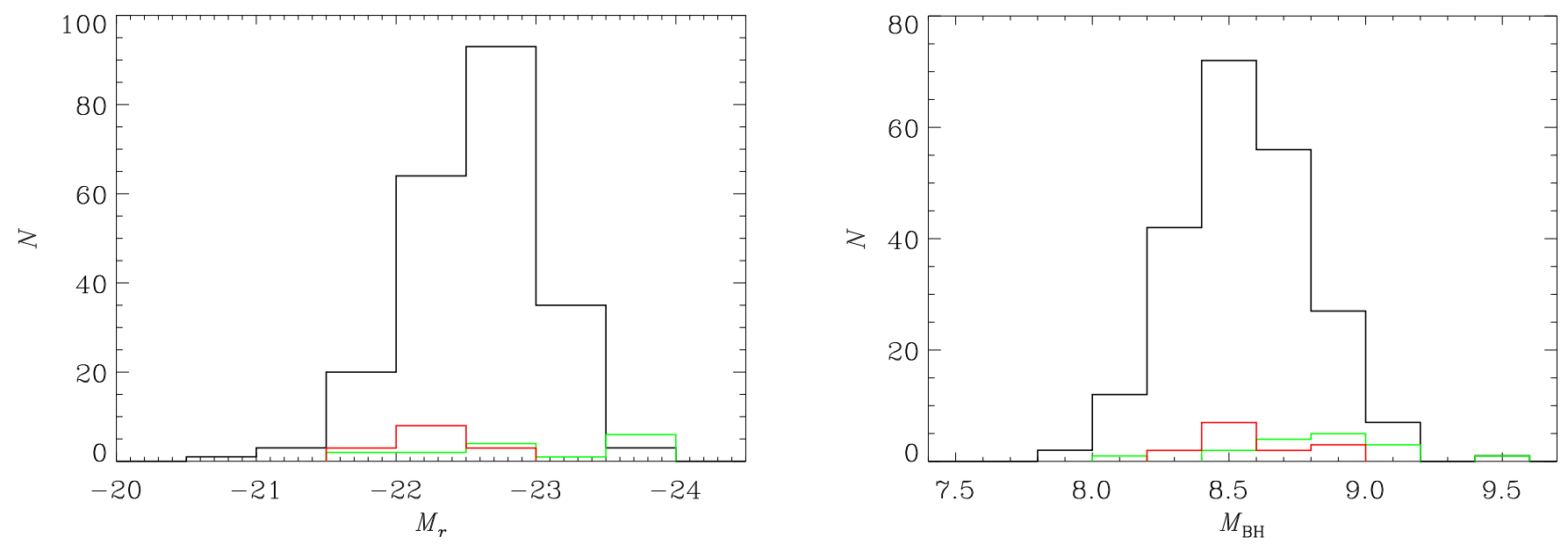

Fig. 4. Distributions of the $r$ band absolute magnitude (left) and black hole masses (right), black for FRICAT, red for the sFRICAT, and green for the 3C-FRIs.

spectroscopically because some of the diagnostic emission lines cannot be measured in their spectra (see Tables B.1 and B.2); based on the criteria used by Best \& Heckman (2012) their radio emission is powered by an AGN. Furthermore, Baldi \& Capetti (2010) show that the spectroscopically unclassified objects likely belong to the class of LEG, but with an even lower contrast of the AGN against the host galaxy emission.

The distribution of absolute magnitude of the FRICAT hosts covers the range $-21 \gtrsim M_{r} \gtrsim-24$ with a maximum at $M_{r} \sim$ -22.5 (see Fig. 4, left panel). The distribution of black hole masses (Fig. 4, right panel) covers the range $8.0 \lesssim \log M_{\mathrm{BH}} \lesssim$ $9.5 M_{\odot}$, peaking at $\sim 10^{8.5} M_{\odot}$.

Various diagnostics can be used for a morphological and spectroscopic classification of the hosts.

The concentration index $C_{r}$ is defined as the ratio of the radii including $90 \%$ and $50 \%$ of the light in the $r$ band, respectively. Early-type galaxies (ETGs) have higher values of $C_{r}$ than late-type galaxies. Two thresholds have been suggested to define ETGs: a more conservative value at $C_{r} \gtrsim 2.86$ (Nakamura et al. 2003; Shen et al. 2003) and a more relaxed selection at $C_{r} \gtrsim 2.6$ (Strateva et al. 2001; Kauffmann et al. 2003; Bell et al. 2003). Bernardi et al. (2010) found that the second threshold of the concentration index corresponds to a mix of $\mathrm{E}+\mathrm{S} 0+\mathrm{Sa}$ types, while the first mainly selects ellipticals galaxies, removing the majority of Sas, but also some Es and SOs.

The Dn(4000) spectroscopic index is defined according to Balogh et al. (1999) as the ratio between the flux density measured on the "red" side of the Ca II break (4000-4100 ̊) and that on the "blue" side (3850-3950 $\AA$ ). Low redshift $(z<0.1)$ red galaxies show $\operatorname{Dn}(4000)=1.98 \pm 0.05$, which is a value that decreases to $=1.95 \pm 0.05$ for $0.1<z<0.15$ galaxies (Capetti \& Raiteri 2015). The presence of young stars or of nonstellar emission reduces the Dn(4000) index.

In Fig. 5 we show the concentration index $C_{r}$ versus the Dn(4000) index (left panel) and versus $M_{\mathrm{BH}}$ (right panel) for the FRICAT sources. The vast majority of the hosts lie in the region of high $C_{r}$ and $\operatorname{Dn}(4000)$ values, indicating that they are red ETGs. There are only a few exceptions: FRICAT $0735+4158$ has a low concentration index $\left(C_{r}=2.29\right)$, but this is due to the presence of two compact sources close to the host center. FRICAT 1053+4929, FRICAT 1428+4240, and FRICAT 1518+0613 instead have a low Dn(4000) index, 1.3; their spectra are rich in absorption lines, suggesting a dilution from nonstellar continuum rather than young stars. Indeed all three sources (that we keep in FRICAT) are included in the list of low luminosity BL Lacs compiled by Capetti \& Raiteri (2015). 

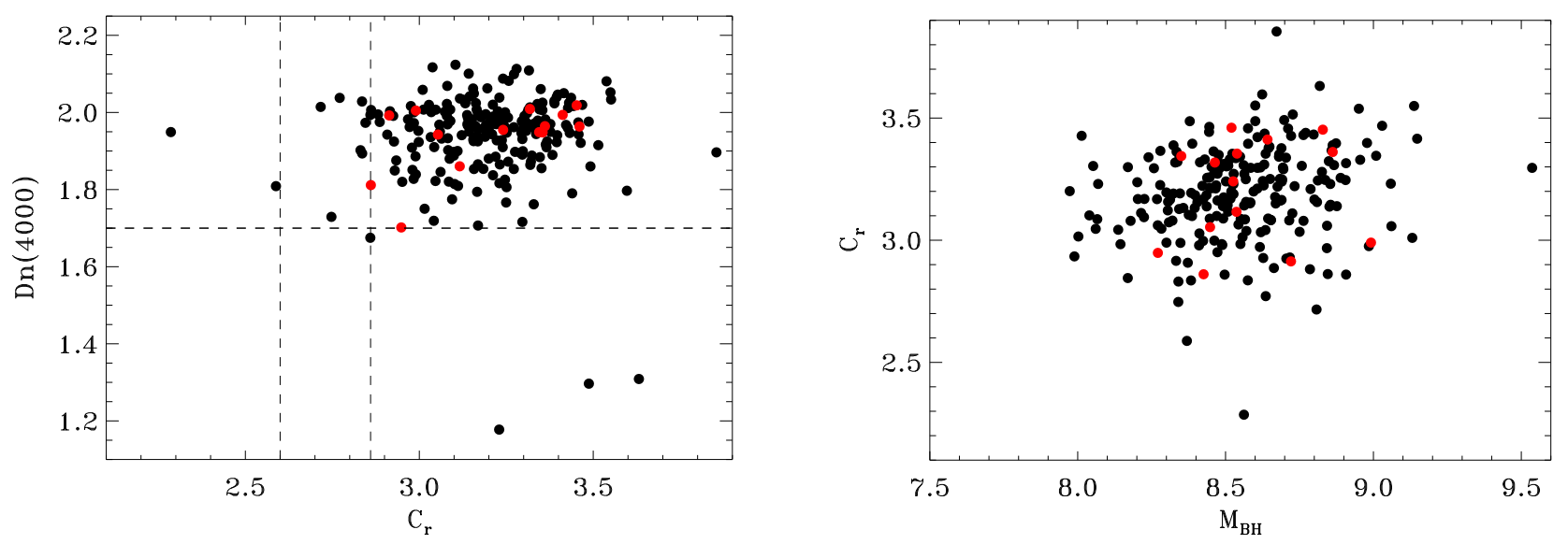

Fig. 5. Left: concentration index $C_{r}$ vs. Dn(4000) index for the FRICAT and the sFRICAT samples (red dots). Right: logarithm of the black hole mass (in solar units) vs. concentration index $C_{r}$.
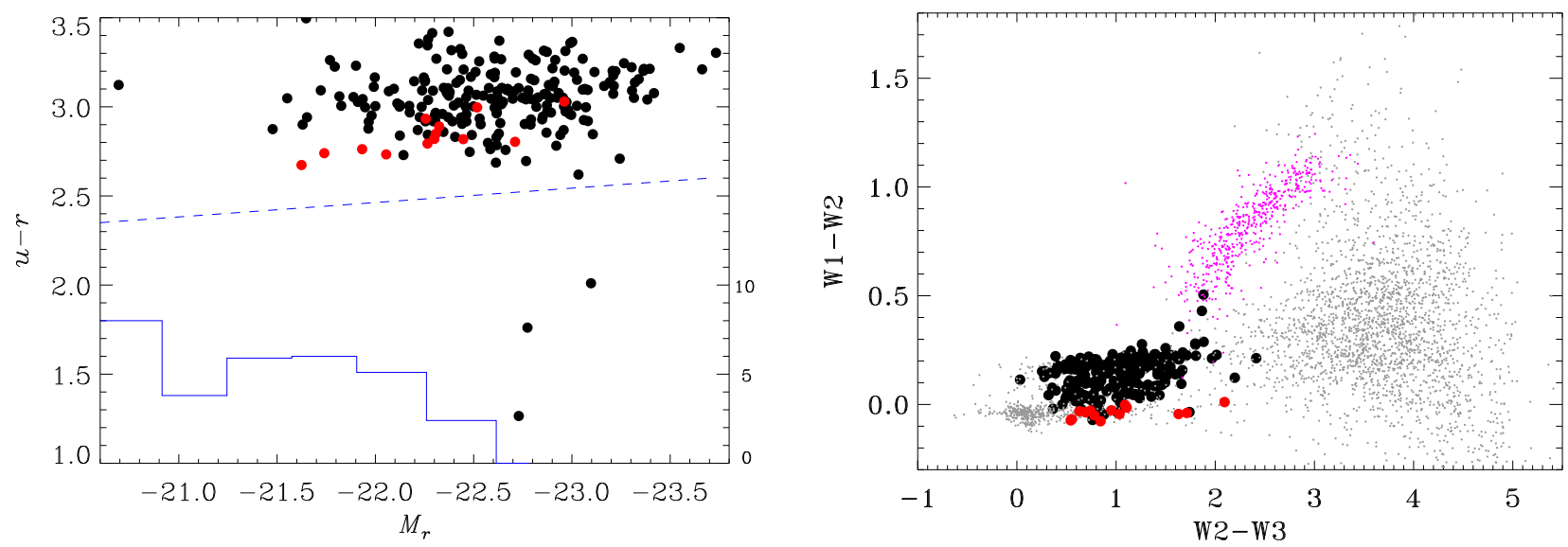

Fig. 6. Left: absolute $r$ band magnitude, $M_{r}$, vs. $u-r$ color for the FRICAT hosts (the red dots represent the sFRICAT sample). The blue histogram on the bottom shows the percentage of blue ETGs (scale on the right axis) from Schawinski et al. (2009). The dashed line separates the "blue" ETG from the red sequence, following their definition. Right: WISE mid-IR colors of the FRICAT hosts compared to those of $\sim 3000$ randomly selected IR sources (gray dots) selected at high Galactic latitudes. We also show the region occupied by the Fermi blazars (purple dots).

The Dn(4000) index refers only to the region covered by the SDSS spectroscopic aperture, $3^{\prime \prime}$ in diameter. In order to explore the global properties of the FRICAT hosts, we also consider the $u-r$ color of the galaxies as a whole. In Fig. 6 we show the $u-r$ color versus the absolute $r$-band magnitude $M_{r}$ of the hosts. With the exception of the three BL Lacs, they are all located above the line separating red and blue ETGs. The fraction of "blue" ETGs (represented as the histogram at the bottom of the figure) decreases with increasing luminosity and these ETGs disappear for $M_{r} \lesssim-22.5$ (Schawinski et al. 2009). The lack of blue ETGs among the FRICAT hosts is relevant; however, their expected number, based on their $M_{r}$ distribution and the "blue" fraction of the general ETGs population, is only 4.3.

The WISE infrared colors further support the passive nature of the FRICAT hosts. In Fig. 6 we show the comparison between the mid-IR colors of FRICAT sources and those of 3000 randomly selected sources (gray circles) at high Galactic latitudes (i.e., $|b|>40^{\circ}$ ). The associations between the FRICAT and the WISE catalog were computed adopting a 3"!3 angular separation, which corresponds to the combination of the typical positional uncertainty of the WISE all sky survey (Wright et al. 2010) and that of the FIRST (D'Abrusco et al. 2014). Fiftyseven of them are undetected in the W3 band and these objects are not reproduced in the figure. In the same figure we also report the mid-IR colors of the Fermi blazars for reference of
WISE sources whose IR emission is dominated by nonthermal radiation (Massaro et al. 2011; D'Abrusco et al. 2012). FRICAT sources appear to have mid-IR colors mostly dominated by their host galaxies (they fall in the same region as elliptical galaxies; Wright et al. 2010) and not contaminated by the emission of their jets. Only the three BL Lacs have $W 2-W 3>0.3$ and they are located at the onset of the sequence defined by the more luminous objects of this class (Massaro et al. 2012).

\subsection{Radio properties}

The distribution of radio luminosity at $1.4 \mathrm{GHz}$ of the FRICAT covers the range $L_{1.4}=v_{\mathrm{r}} l_{\mathrm{r}}=\sim 10^{39.5}-10^{41.3} \mathrm{erg} \mathrm{s}^{-1}$ those of the sFRICAT sample instead have $10^{39} \lesssim L_{1.4} \lesssim 10^{40.4} \mathrm{erg} \mathrm{s}^{-1}$. The Fanaroff \& Riley (1974) separation between FR Is and FR IIs translates, with our adopted cosmology and by assuming a spectral index of 0.7 between $178 \mathrm{MHz}$ and $1.4 \mathrm{GHz}$, into $L_{1.4} \sim$ $10^{41.6} \mathrm{erg} \mathrm{s}^{-1}$. All objects included in our sample fall below this threshold, although it must be kept in mind that the power separation between FR Is and FR IIs is sharper at $178 \mathrm{MHz}$ than at higher frequencies (Zirbel \& Baum 1995).

The separation between FR classes is cleaner in the opticalradio luminosity plane (Ledlow \& Owen 1996). Indeed, the bulk of the FRICAT sources lie below the boundary between FR I and FR II reported by Ledlow \& Owen (see left panel Fig. 7) in the region populated by FR I sources. Be aware that we shifted 

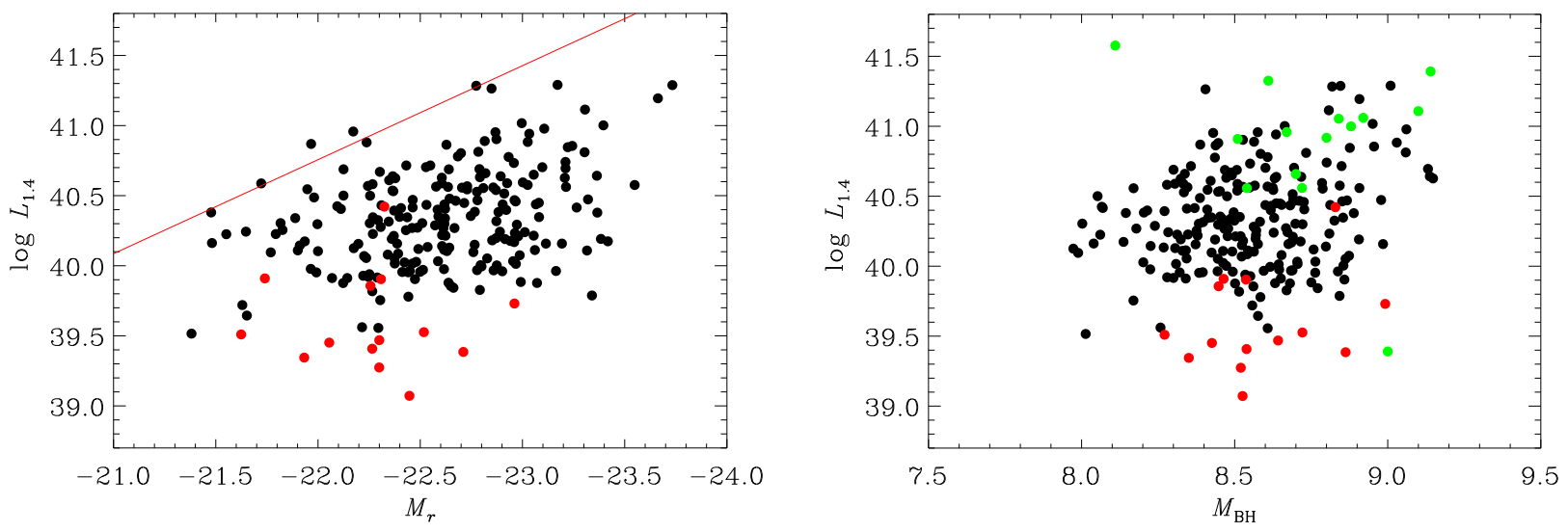

Fig. 7. Left panel: radio luminosity (NVSS) vs. host absolute magnitude, $M_{r}$, for FRICATand sFRICAT (black and red, respectively). The solid line shows the separation between FR I and FR II reported by Ledlow \& Owen (1996) to which we applied a correction of 0.34 mag to account for the different magnitude definition and the color transformation between the SDSS and Cousin systems. Right panel: radio luminosity vs. black hole mass. The green points are the 3C-FR Is.

the dividing line to the right of the diagram to include a correction of $0.12 \mathrm{mag}$ to scale our total host magnitude to the $\mathrm{M}_{24.5}$ used by these authors, and an additional 0.22 mag to convert the Cousin system into the SDSS system (Fukugita et al. 1996). This confirms the indication that more powerful FR Is can be associated with more massive galaxies, while in less luminous hosts the FR I/FR II transition occurs at lower $L_{1.4}$; as a result, a positive trend links $L_{1.4}$ and $M_{\mathrm{r}}$. A similar trend is seen also between $L_{1.4}$ and $M_{\mathrm{BH}}$ (Fig. 7, right panel). This is likely to be driven by the connection between $M_{\mathrm{BH}}$ and the host luminosity (Marconi \& Hunt 2003) combined with the Ledlow \& Owen effect.

FR Is show a large spread in both radio and [O III] line luminosities (see Fig. 8), both quantities spanning over two orders of magnitude. The FR Is of the sFRICAT sample fall generally in the low end of the radio luminosity distribution. Within the same volume $(z<0.05)$, the sources extending to $10<r<30 \mathrm{kpc}$ have a median luminosity that are four times smaller than those with $r>30 \mathrm{kpc}$.

\section{Discussion}

The population of the FRICAT hosts is remarkably uniform. They are all luminous red ETGs, with large black hole masses $\left(M_{\mathrm{BH}} \gtrsim 10^{8} M_{\odot}\right)$, spectroscopically classified as LEGs. All these properties are shared with the hosts of the "small" FR Is and the more powerful 3C-FR Is. We included in the 3C-FRIs sample the 16 radio galaxies with $z<0.3$ and a FR I morphology, according to Buttiglione et al. (2010), and with either a direct $M_{\mathrm{BH}}$ measurement or a published stellar velocity dispersion in the HyperLeda database ${ }^{2}$. More quantitatively, the distributions of $M_{\mathrm{BH}}$ and $M_{r}$ of the FRICAT and sFRICAT samples are not statistically distinguishable, according to the Kolmogoroff-Smirnov test. A small difference might instead emerge when considering the 3C-FRIs hosts. This latter sample has a median $M_{\mathrm{BH}}$ that is a factor of 1.9 higher with respect to the FRICAT (and they are 0.2 mag brighter). As discussed in the previous section, this might be the manifestation of the Ledlow \& Owen effect. Nonetheless, the null hypothesis that they are drawn from different populations cannot be rejected at a $3 \sigma$ confidence level.

Even though the hosts of three FR Is samples are very similar, the FRICAT sources show a very different properties with

\footnotetext{
2 http://leda.univ-lyon $1 . \mathrm{fr} /$
}

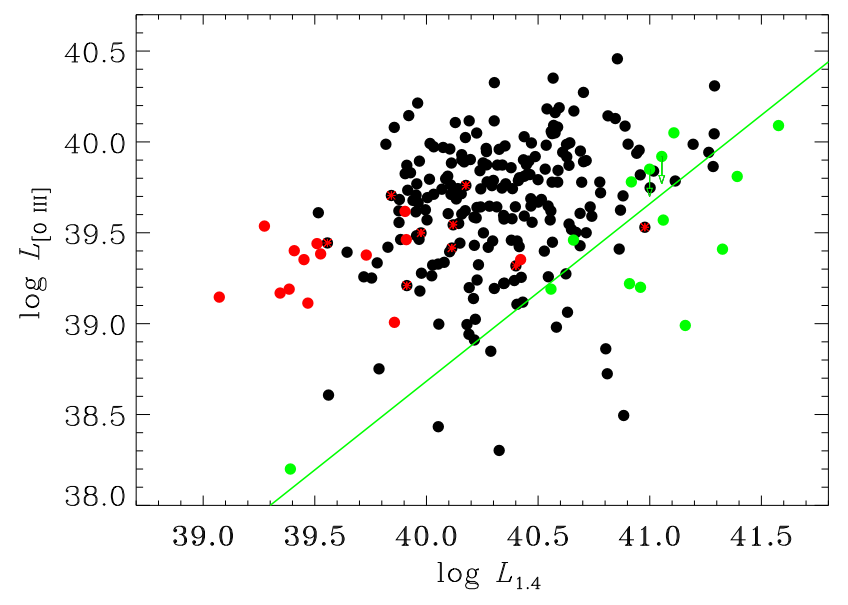

Fig. 8. Radio (NVSS) vs. [O III] line luminosity of the FRICAT, sFRICAT, and 3C-FR I samples (black, red, and green points, respectively). The FRICAT sources with $z<0.05$ are represented as black dots with a red asterisk superimposed. The green line shows the linear correlation between these two quantities derived from the FR Is of the 3C sample from (Buttiglione et al. 2010).

respect to what is seen in the $3 \mathrm{C}$ sample for the connection between emission lines and radio luminosities.

The 3C-FRIs show a positive trend between the line and radio luminosities with a slope consistent with unity (e.g., Buttiglione et al. 2010). This indicates that a constant fraction of the AGN power, as measured from the emission lines, is converted into radio emission. Buttiglione et al. show that the same result, although with a different normalization, is found when considering the $3 \mathrm{C}$-FR II radio galaxies.

Conversely, no correlation between $L_{1.4}$ and $L_{[\mathrm{O} \text { III] }}$ can be seen for the FRICAT where, at a given line luminosity, the radio luminosities span over two orders of magnitude. Similar to what is seen in the 3C sample, it appears that no source has a $\left.L_{1.4} / L_{[\mathrm{O}} \mathrm{III}\right]$ ratio exceeding $\sim 100$, producing the scarcely populated region in the bottom right portion of this diagram; but objects are found with much lower ratios down to $\left.L_{1.4} / L_{[\mathrm{O}} \mathrm{III}\right] \sim 0.5$. Furthermore, the radio luminosity grows, not surprisingly, when the size of the radio source increases. Less obviously, the FR Is in the "small" sample have a lower (by a factor of $\sim 3$ ) median ratio between line and radio luminosity.

Apparently, the high flux threshold used for the selection of the $3 \mathrm{C}$ sources favored the inclusion of radio galaxies with high 
ratios between radio and optical luminosity. A much larger population of FR Is emerges when lowering the radio flux limit by three orders of magnitude. The connection between radio and line luminosity disappears. The spectroscopic and host properties of the FR I hosts rule out the possibility of a substantial contribution to the [O III] line by star formation that might compromise our ability to reveal this trend, if indeed this was present. The broad distribution of $L_{1.4} / L_{[\mathrm{O}}$ III] ratio indicates a corresponding broad range of conversion of AGN bolometric power into radio emission.

\section{Summary and conclusions}

We built a catalog of 219 FR I radio galaxies, called FRICAT, selected from the Best \& Heckman (2012) sample, and obtained by combining the SDSS, NVSS, and FIRST surveys.

The FR I classification is purely morphological and based on the visual inspection of the FIRST radio images. We included the sources in which the radio emission reaches a distance of at least $30 \mathrm{kpc}$ from the host (restricting the analysis to those with redshift $z<0.15$ ). We adopted rather strict criteria for a positive FR I classification; we selected only sources showing one-sided or two-sided jets in which the surface brightness is generally decreasing along its whole length, lacking of any brightness enhancement at the jet end, i.e., with an edge-darkened structure The resulting FRICAT catalog comprises 219 objects. A second sample of 14 objects, sFRICAT, extends the selection to smaller FR Is by including sources with $10<r<30 \mathrm{kpc}$ and $z<0.05$.

These samples have a high level of completeness ( 90\%) in both their radio and optical selection. As such, they can be used to study, for example, their radio and bivariate radio/optical luminosity functions. One should nonetheless bear in mind the morphological selection criteria adopted and that the completeness limit in the radio band is $\sim 50 \mathrm{mJy}$. These are well suited for our purposes but it will be certainly interesting to explore the connection of the FRICAT with the remaining $~ 500$ extended radio galaxies we did not include in our analysis.

The FRICAT hosts are remarkably homogeneous, as they are all luminous red ETGs with large black hole masses that are spectroscopically classified as LEGs. All these properties are shared by the hosts of more powerful FR Is in the 3C sample. They do not show significant differences from the point of view of their colors with respect to the general population of massive ETGs. The presence of an active nucleus (and its level of activity) does not appear to affect the hosts of FR Is.

The FRICAT sources differ from the 3C-FRIs for the connection between emission lines and radio luminosities. While in 3C-FRIs the line and radio luminosities are correlated (suggesting that a constant fraction of the AGN power is converted into radio emission) these two quantities are unrelated in the FRICAT. We argue that the line/radio correlation is the result of a selection bias because of the high flux threshold of the $3 \mathrm{C}$ sources that favors the inclusion of radio galaxies with high ratios between radio and optical luminosity. Baldi \& Capetti (2009) reached a similar conclusion from the comparison of the $3 \mathrm{C}$ objects with the radio galaxies associated with nearby $(z \lesssim 0.01)$ optically luminous ETGs.

The 3C-FRIs represent the tip of the iceberg of a much larger and diverse population of FR Is. This result highlights the importance of exploring a broader (and larger) population of FR Is. Several other issues, such as those listed in the Introduction, can now be addressed by using FRICAT. In particular we will explore in two forthcoming papers the environment of FRICAT and how they are related to the class of the compact FR 0 radio sources (Baldi et al. 2015).
Acknowledgements. F.M. gratefully acknowledges the financial support of the Programma Giovani Ricercatori - Rita Levi Montalcini - Rientro dei Cervelli (2012) awarded by the Italian Ministry of Education, Universities and Research (MIUR). Part of this work is based on the NVSS (NRAO VLA Sky Survey): The National Radio Astronomy Observatory is operated by Associated Universities, Inc., under contract with the National Science Foundation. This publication makes use of data products from the Wide-field Infrared Survey Explorer, which is a joint project of the University of California, Los Angeles, and the Jet Propulsion Laboratory/California Institute of Technology, funded by the National Aeronautics and Space Administration. This research made use of the NASA/IPAC Infrared Science Archive and Extragalactic Database (NED), which are operated by the Jet Propulsion Laboratory, CaliforniaInstitute of Technology, under contract with the National Aeronautics and Space Administration. We acknowledge the usage of the HyperLeda database (http://leda. univ-lyon1.fr). Funding for SDSS-III has been provided by the Alfred P. Sloan Foundation, the Participating Institutions, the National Science Foundation, and the US Department of Energy Office of Science. The SDSS-III web site is http://www.sdss3.org/. SDSS-III is managed by the Astrophysical Research Consortium for the Participating Institutions of the SDSS-III Collaboration, including the University of Arizona, the Brazilian Participation Group, Brookhaven National Laboratory, University of Cambridge, Carnegie Mellon University, University of Florida, the French Participation Group, the German Participation Group, Harvard University, the Instituto de Astrofisica de Canarias, the Michigan State/Notre Dame/JINA Participation Group, Johns Hopkins University, Lawrence Berkeley National Laboratory, Max Planck Institute for Astrophysics, Max Planck Institute for Extraterrestrial Physics, New Mexico State University, New York University, Ohio State University, Pennsylvania State University, University of Portsmouth, Princeton University, the Spanish Participation Group, University of Tokyo, University of Utah, Vanderbilt University, University of Virginia, University of Washington, and Yale University.

\section{References}

Abazajian, K. N., Adelman-McCarthy, J. K., Agüeros, M. A., et al. 2009, ApJS, 182,543

Baldi, R. D., \& Capetti, A. 2009, A\&A, 508, 603

Baldi, R. D., \& Capetti, A. 2010, A\&A, 519, A48

Baldi, R. D., Capetti, A., \& Giovannini, G. 2015, A\&A, 576, A38

Balogh, M. L., Morris, S. L., Yee, H. K. C., Carlberg, R. G., \& Ellingson, E. 1999, ApJ, 527, 54

Becker, R. H., White, R. L., \& Helfand, D. J. 1995, ApJ, 450, 559

Bell, E. F., McIntosh, D. H., Katz, N., \& Weinberg, M. D. 2003, ApJS, 149, 289

Bennett, A. S. 1962, MmRAS, 68, 163

Bernardi, M., Shankar, F., Hyde, J. B., et al. 2010, MNRAS, 404, 2087

Best, P. N., \& Heckman, T. M. 2012, MNRAS, 421, 1569

Best, P. N., Kauffmann, G., Heckman, T. M., et al. 2005, MNRAS, 362, 25

Brinchmann, J., Charlot, S., White, S. D. M., et al. 2004, MNRAS, 351, 1151

Buttiglione, S., Capetti, A., Celotti, A., et al. 2010, A\&A, 509, A6

Capetti, A., \& Raiteri, C. M. 2015, A\&A, 580, A73

Colla, G., Fanti, C., Fanti, R., et al. 1975, A\&AS, 20, 1

Condon, J. J., Cotton, W. D., Greisen, E. W., et al. 1998, AJ, 115, 1693

D'Abrusco, R., Massaro, F., Ajello, M., et al. 2012, ApJ, 748, 68

D'Abrusco, R., Massaro, F., Paggi, A., et al. 2014, ApJS, 215, 14

Fanaroff, B. L., \& Riley, J. M. 1974, MNRAS, 167, 31

Fanti, R., Gioia, I., Lari, C., \& Ulrich, M. H. 1978, A\&AS, 34, 341

Fukugita, M., Ichikawa, T., Gunn, J. E., et al. 1996, AJ, 111, 1748

Kauffmann, G., Heckman, T. M., White, S. D. M., et al. 2003, MNRAS, 341, 33

Ledlow, M. J., \& Owen, F. N. 1996, AJ, 112, 9

Marconi, A., \& Hunt, L. K. 2003, ApJ, 589, L21

Massaro, F., D’Abrusco, R., Ajello, M., Grindlay, J. E., \& Smith, H. A. 2011, ApJ, 740, L48

Massaro, F., D’Abrusco, R., Tosti, G., et al. 2012, ApJ, 750, 138

Montero-Dorta, A. D., \& Prada, F. 2009, MNRAS, 399, 1106

Nakamura, O., Fukugita, M., Yasuda, N., et al. 2003, AJ, 125, 1682

Owen, F. N., \& Rudnick, L. 1976, ApJ, 205, L1

Planck Collaboration XIII. 2016, A\&A, 594, A13

Rudnick, L., \& Owen, F. N. 1977, AJ, 82, 1

Schawinski, K., Lintott, C., Thomas, D., et al. 2009, MNRAS, 396, 818

Shen, S., Mo, H. J., White, S. D. M., et al. 2003, MNRAS, 343, 978

Strateva, I., Ivezić, Ž., Knapp, G. R., et al. 2001, AJ, 122, 1861

Strauss, M. A., Weinberg, D. H., Lupton, R. H., et al. 2002, AJ, 124, 1810

Tremaine, S., Gebhardt, K., Bender, R., et al. 2002, ApJ, 574, 740

Tremonti, C. A., Heckman, T. M., Kauffmann, G., et al. 2004, ApJ, 613, 898

Wright, E. L., Eisenhardt, P. R. M., Mainzer, A. K., et al. 2010, AJ, 140, 1868

Zehavi, I., Blanton, M. R., Frieman, J. A., et al. 2002, ApJ, 571, 172

Zirbel, E. L., \& Baum, S. A. 1995, ApJ, 448, 521 
A. Capetti et al.: FRICAT: A FIRST catalog of FR I radio galaxies

\section{Appendix A: FIRST images of the 219 FRICAT sources}
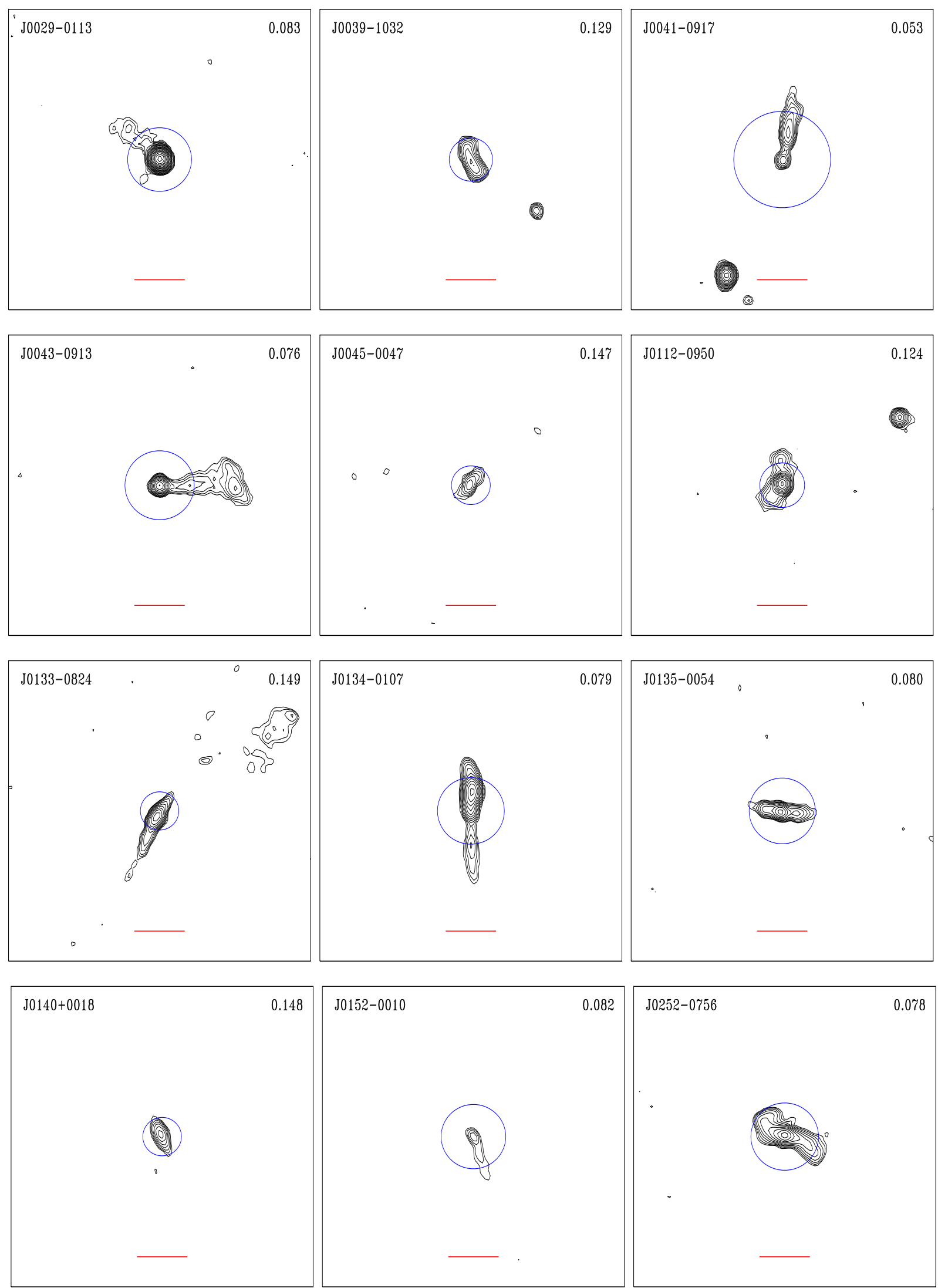

Fig. A.1. Images of the FR Is selected. Contours, corrected for cosmological effects to a redshift of $z=0.15$, are drawn starting from $0.45 \mathrm{mJy} / \mathrm{beam}$ and increase with a geometric progression with a common ratio of $\sqrt{2}$. The field of view is $3^{\prime} \times 3^{\prime}$; the red tick at the bottom is $30^{\prime \prime}$ long. The blue circle is centered on the host galaxy and has a radius of $30 \mathrm{kpc}$. The source ID and redshift are reported in the upper corners. 
A\&A 598, A49 (2017)
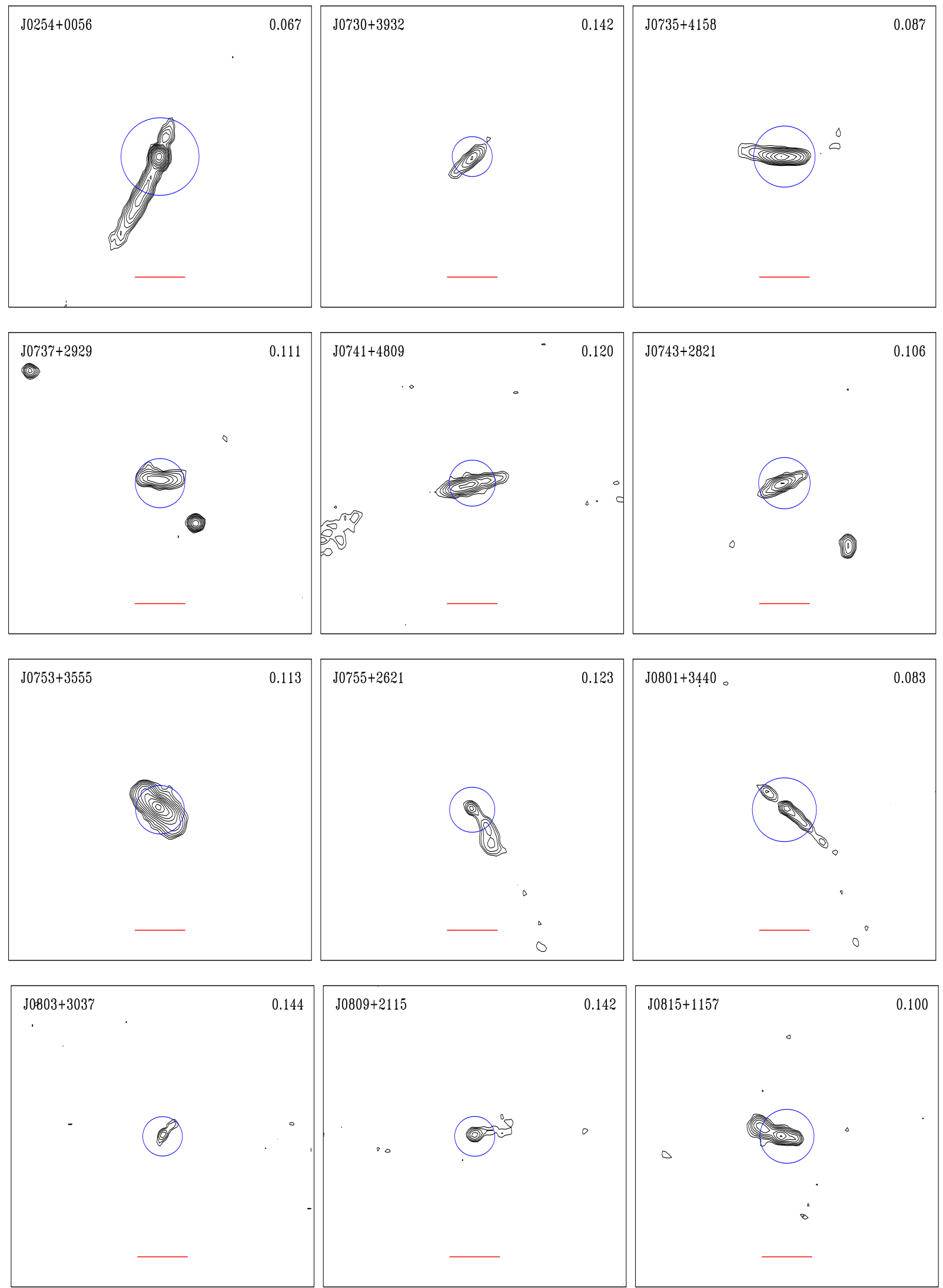

Fig. A.1. continued. 
A. Capetti et al.: FRICAT: A FIRST catalog of FR I radio galaxies
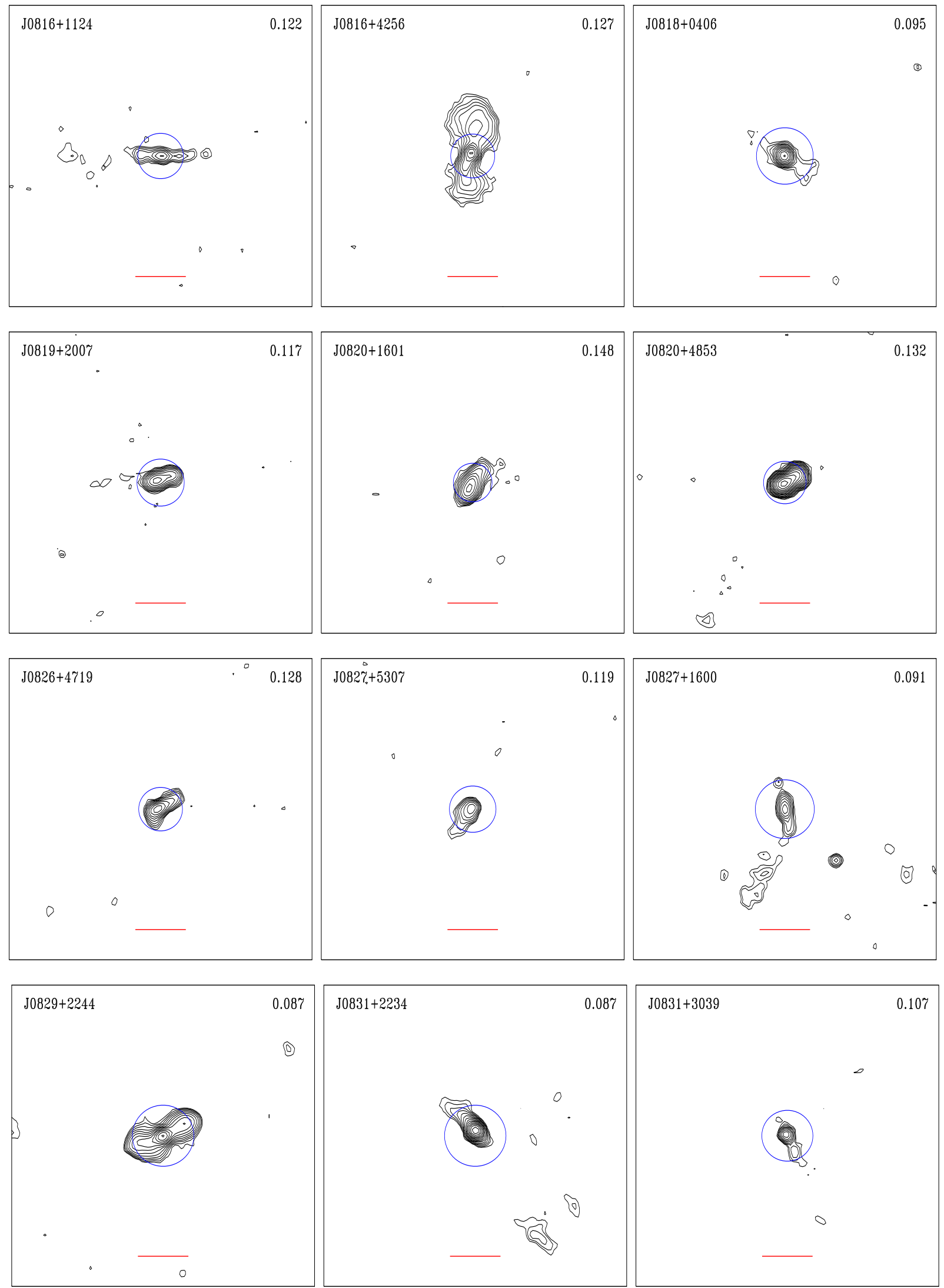

Fig. A.1. continued. 
A\&A 598, A49 (2017)
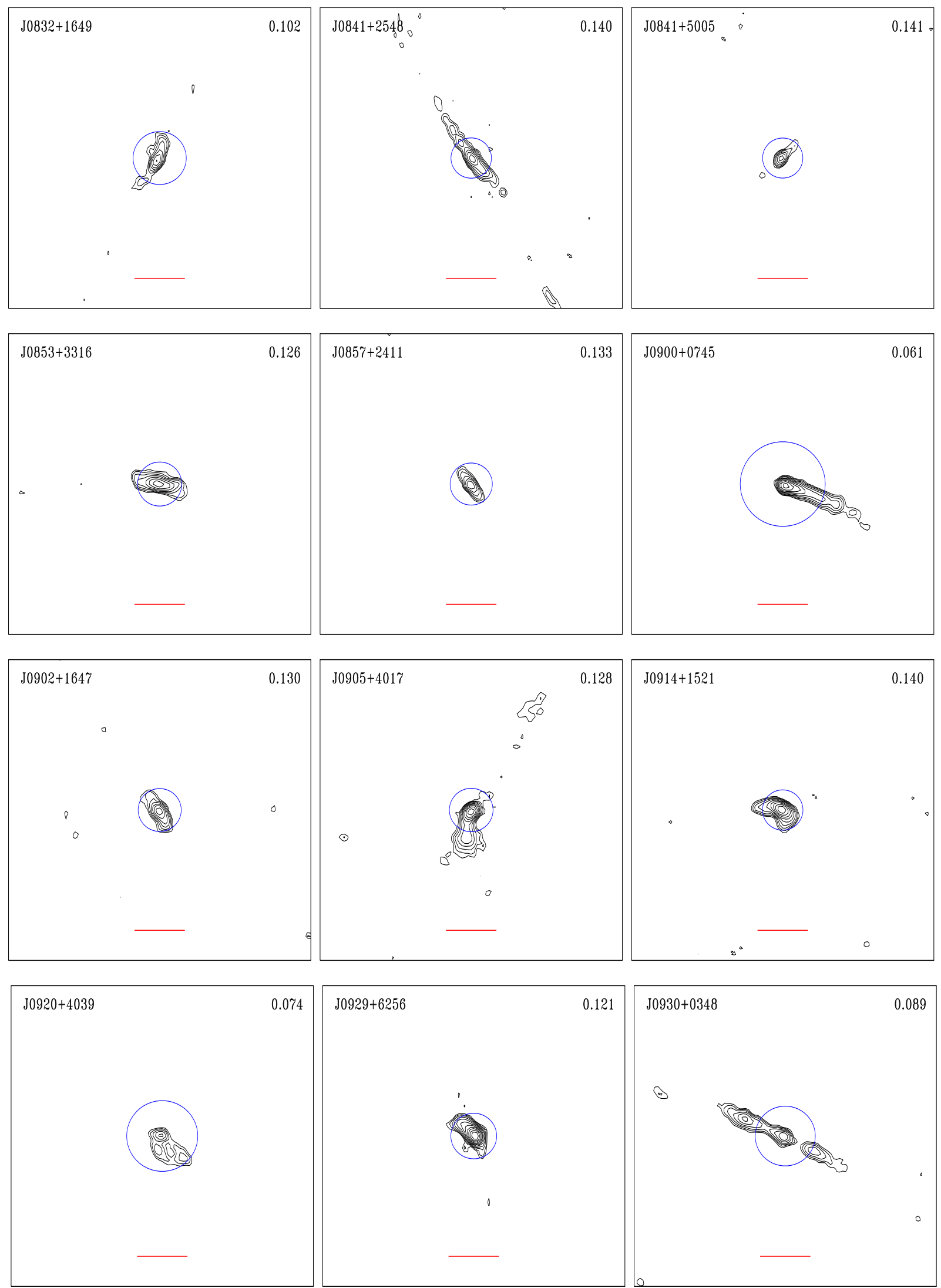

Fig. A.1. continued. 
A. Capetti et al.: FRICAT: A FIRST catalog of FR I radio galaxies
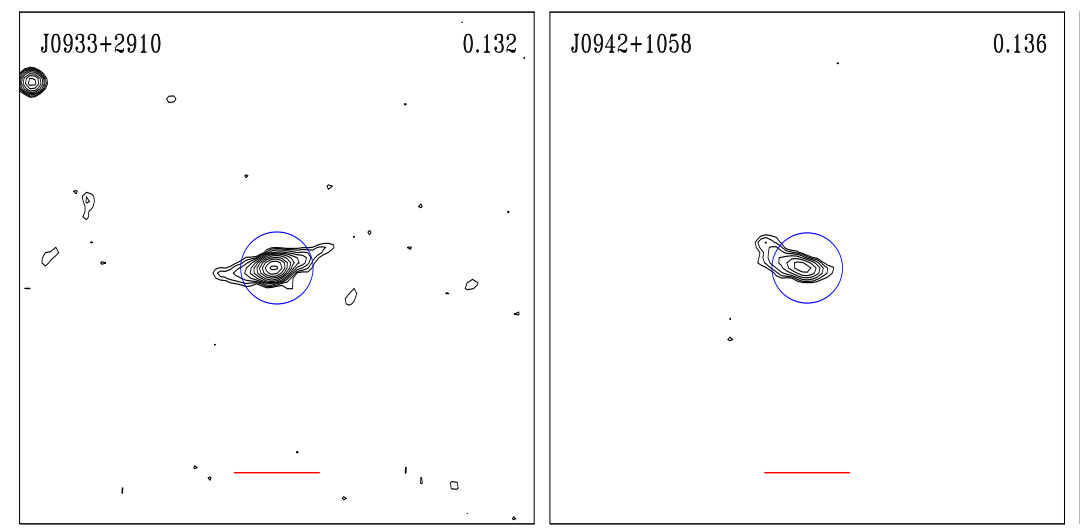

J0943+3341

0.131
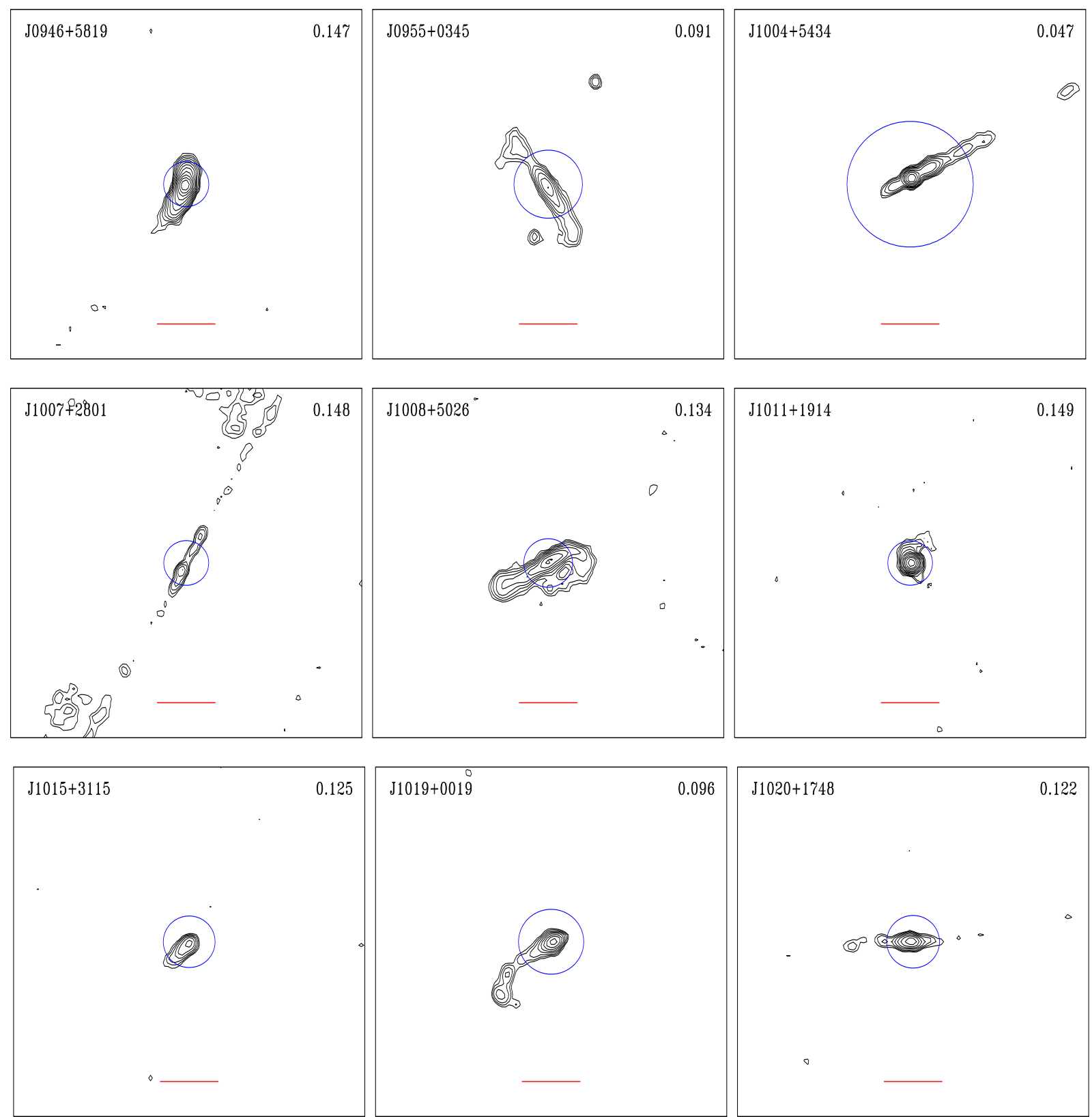

Fig. A.1. continued. 
A\&A 598, A49 (2017)
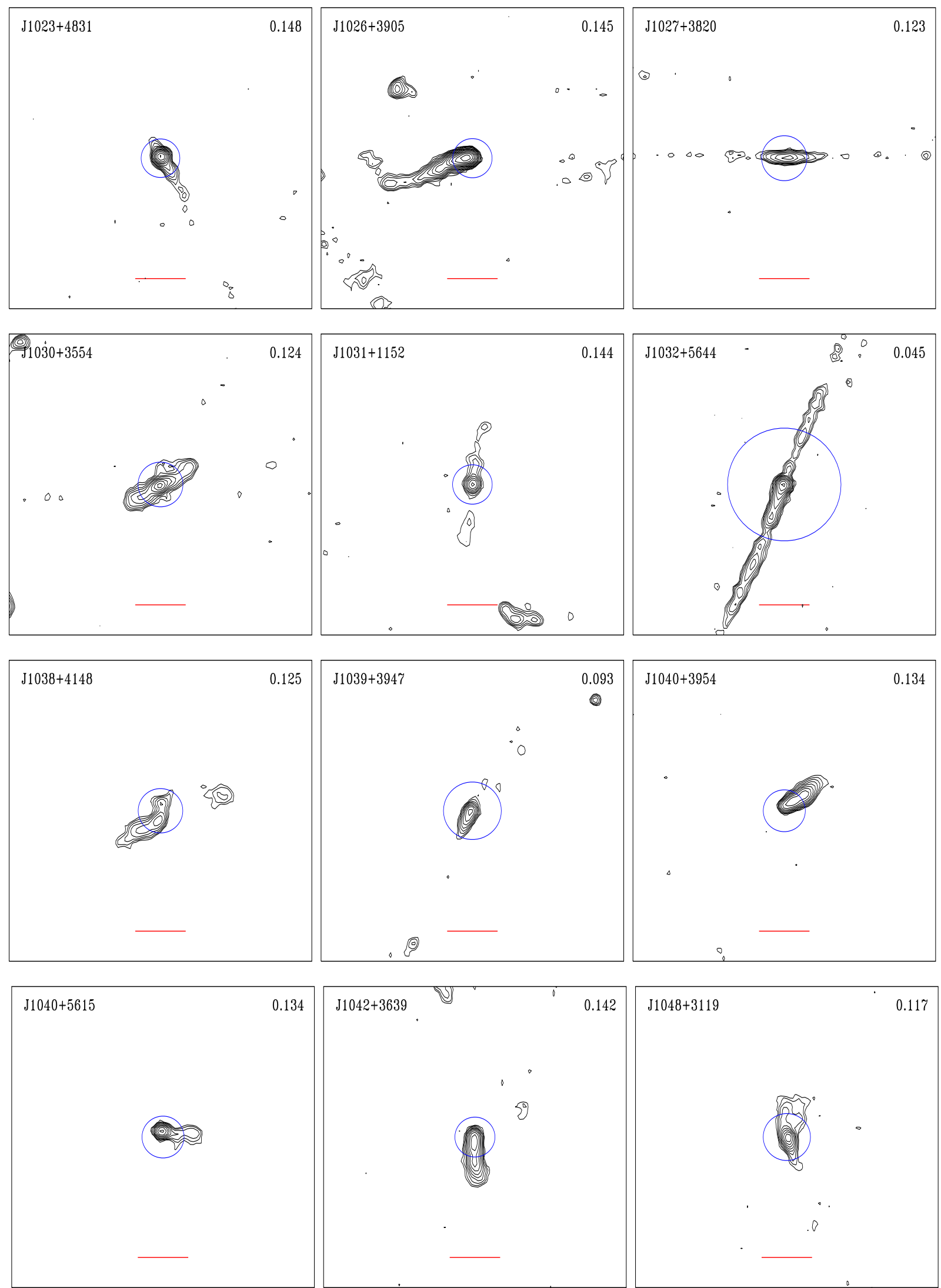

Fig. A.1. continued. 
A. Capetti et al.: FRICAT: A FIRST catalog of FR I radio galaxies
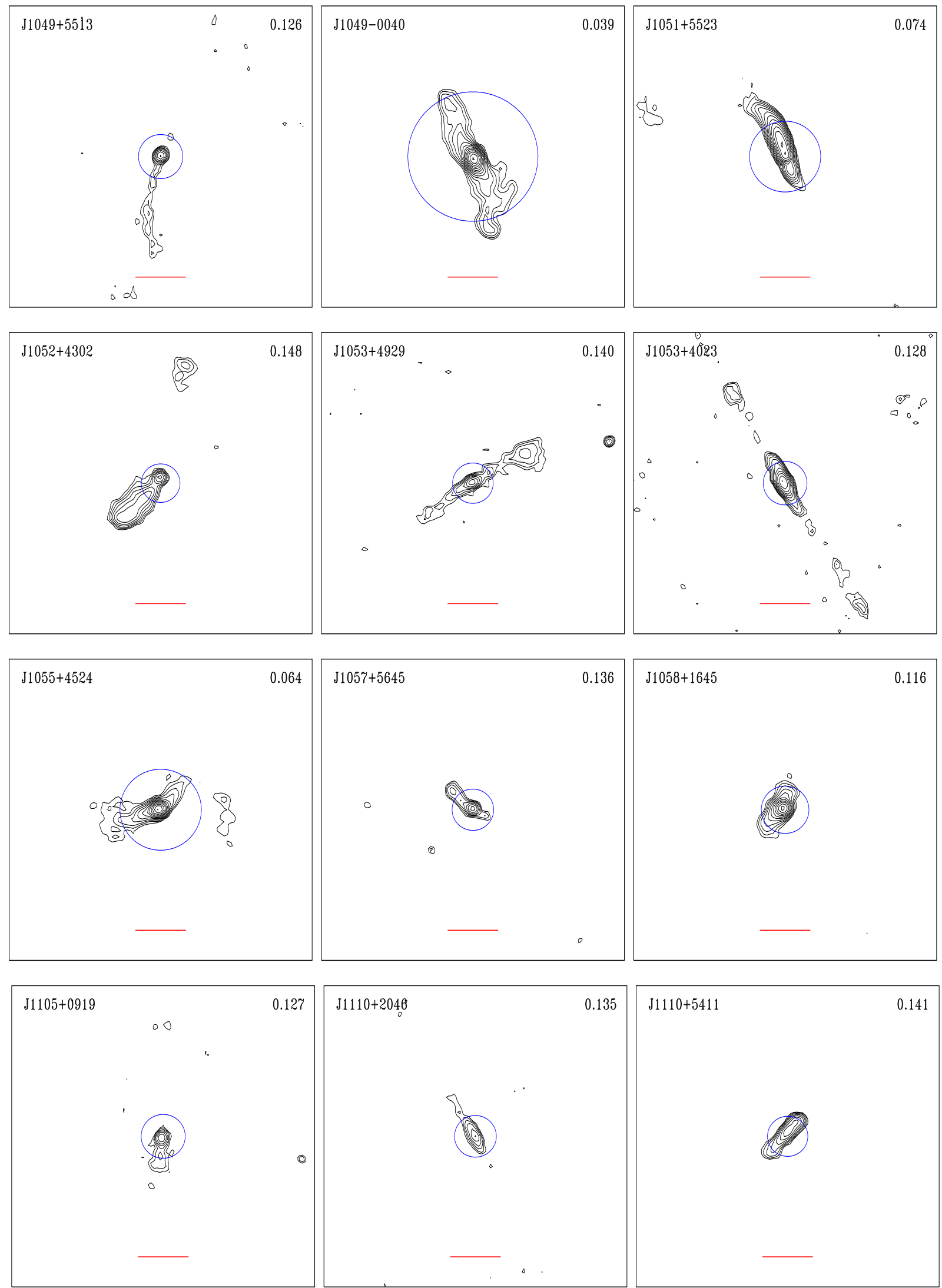

Fig. A.1. continued. 
A\&A 598, A49 (2017)
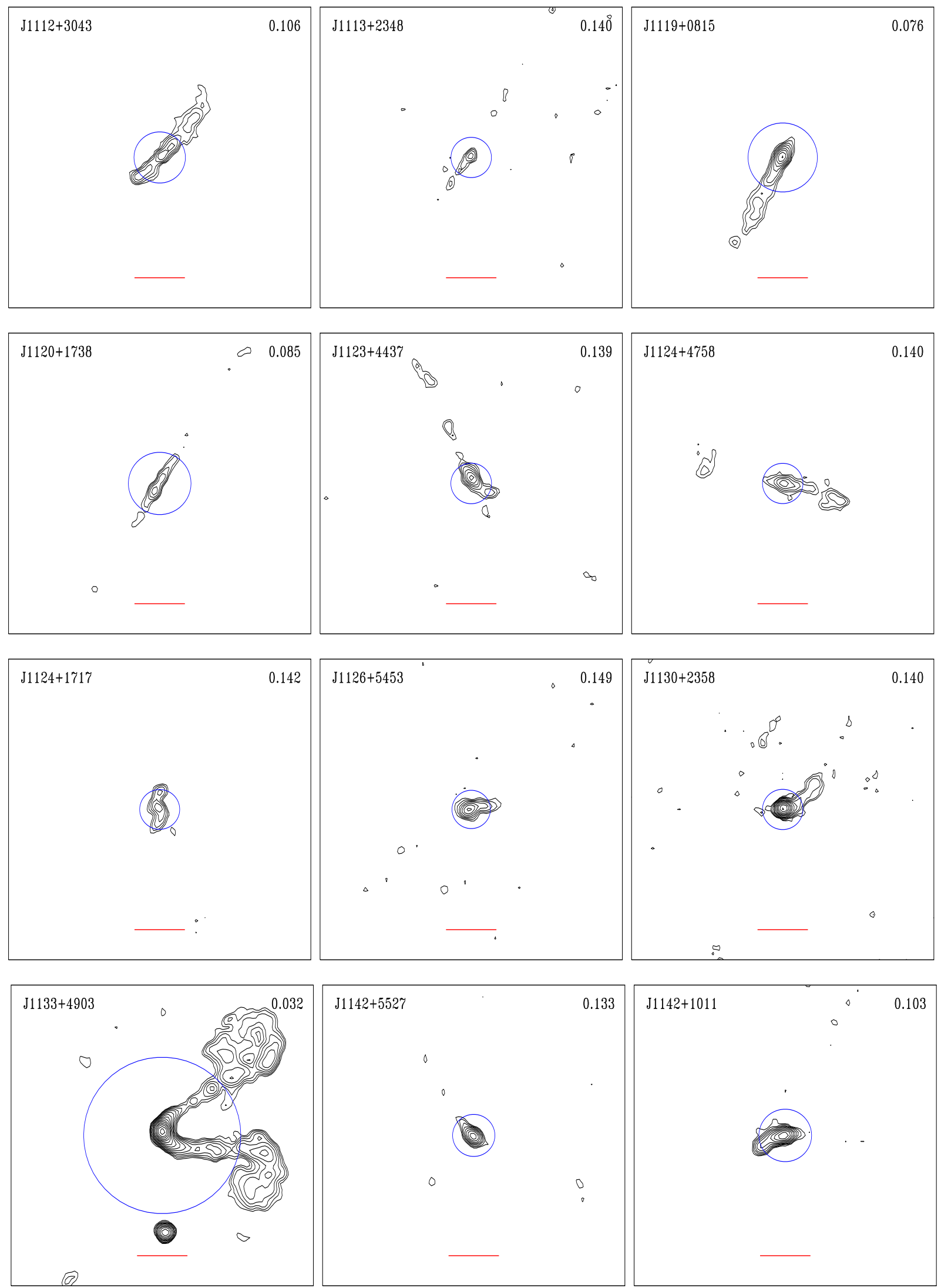

Fig. A.1. continued. 
A. Capetti et al.: FRICAT: A FIRST catalog of FR I radio galaxies
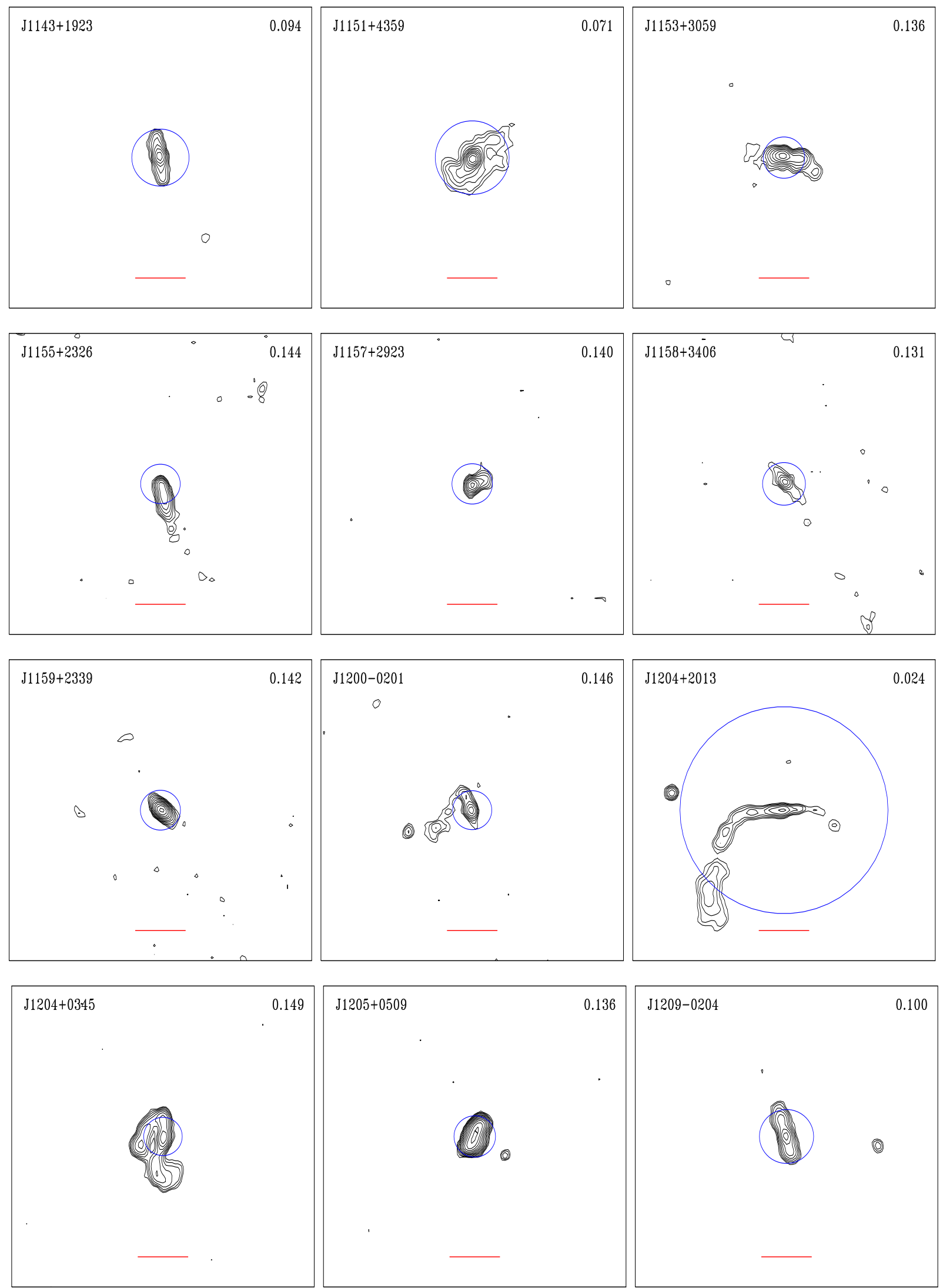

Fig. A.1. continued. 
A\&A 598, A49 (2017)
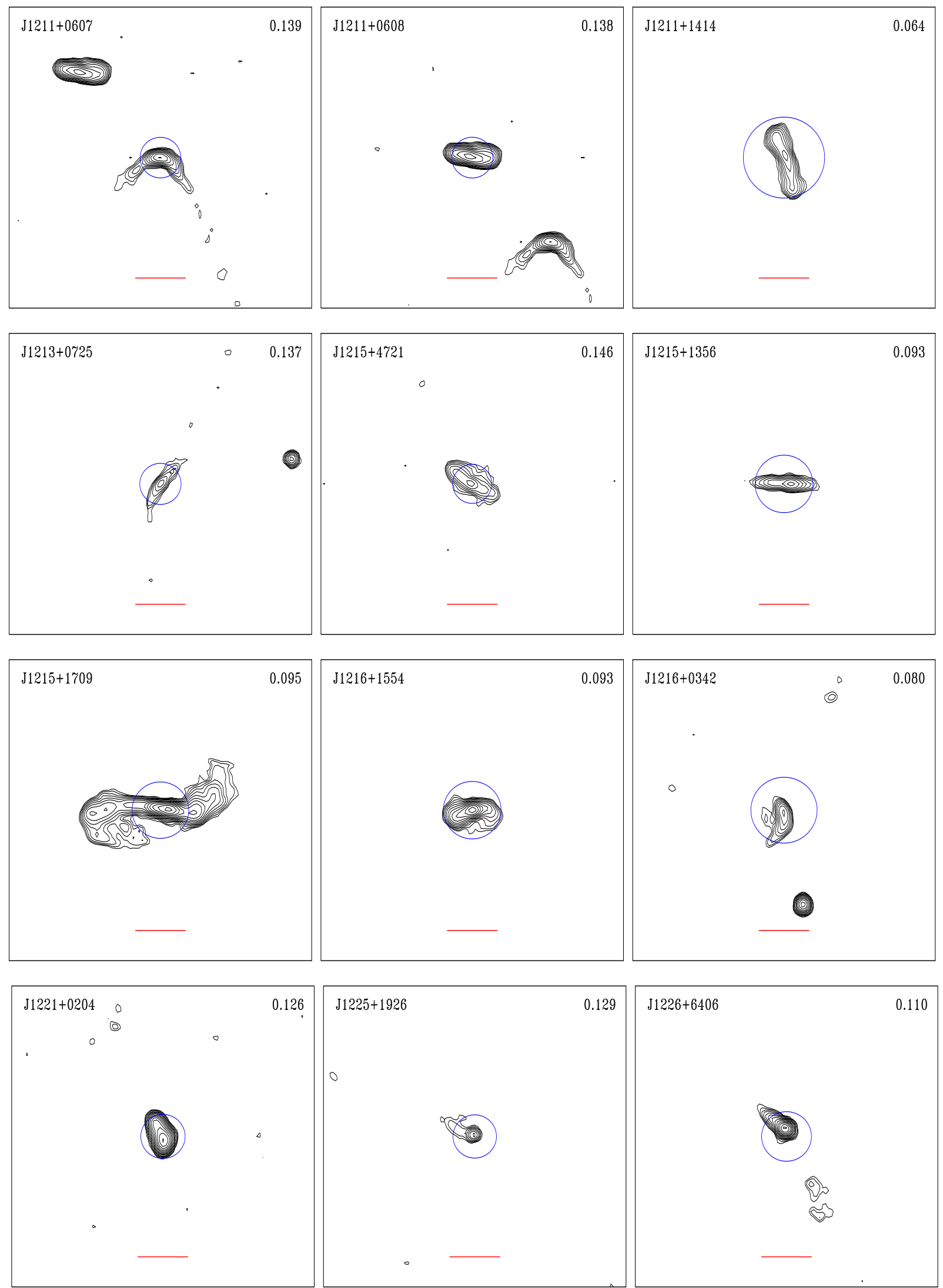

Fig. A.1. continued. 
A. Capetti et al.: FRICAT: A FIRST catalog of FR I radio galaxies
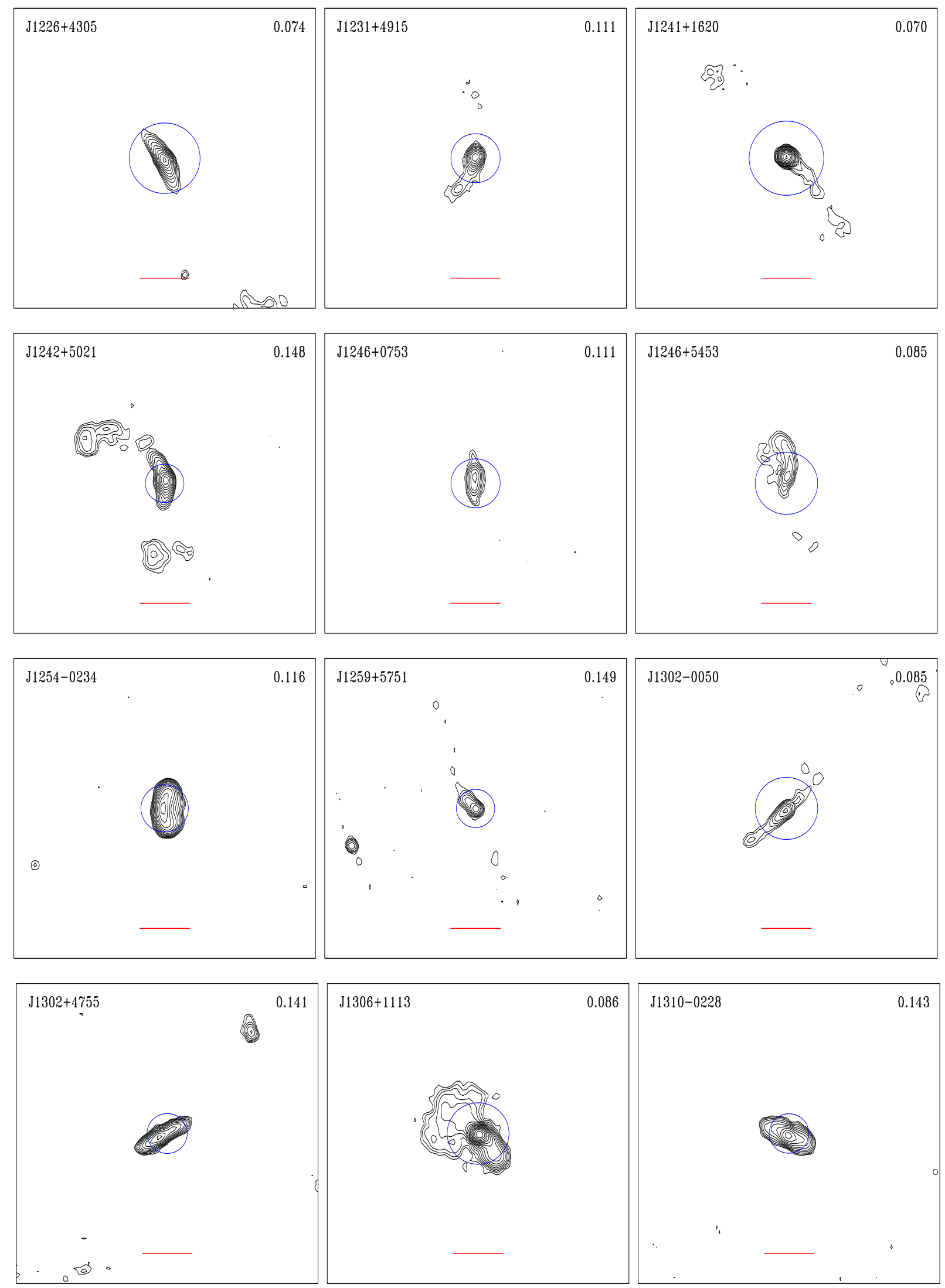

Fig. A.1. continued.

A49, page 19 of 32 
A\&A 598, A49 (2017)
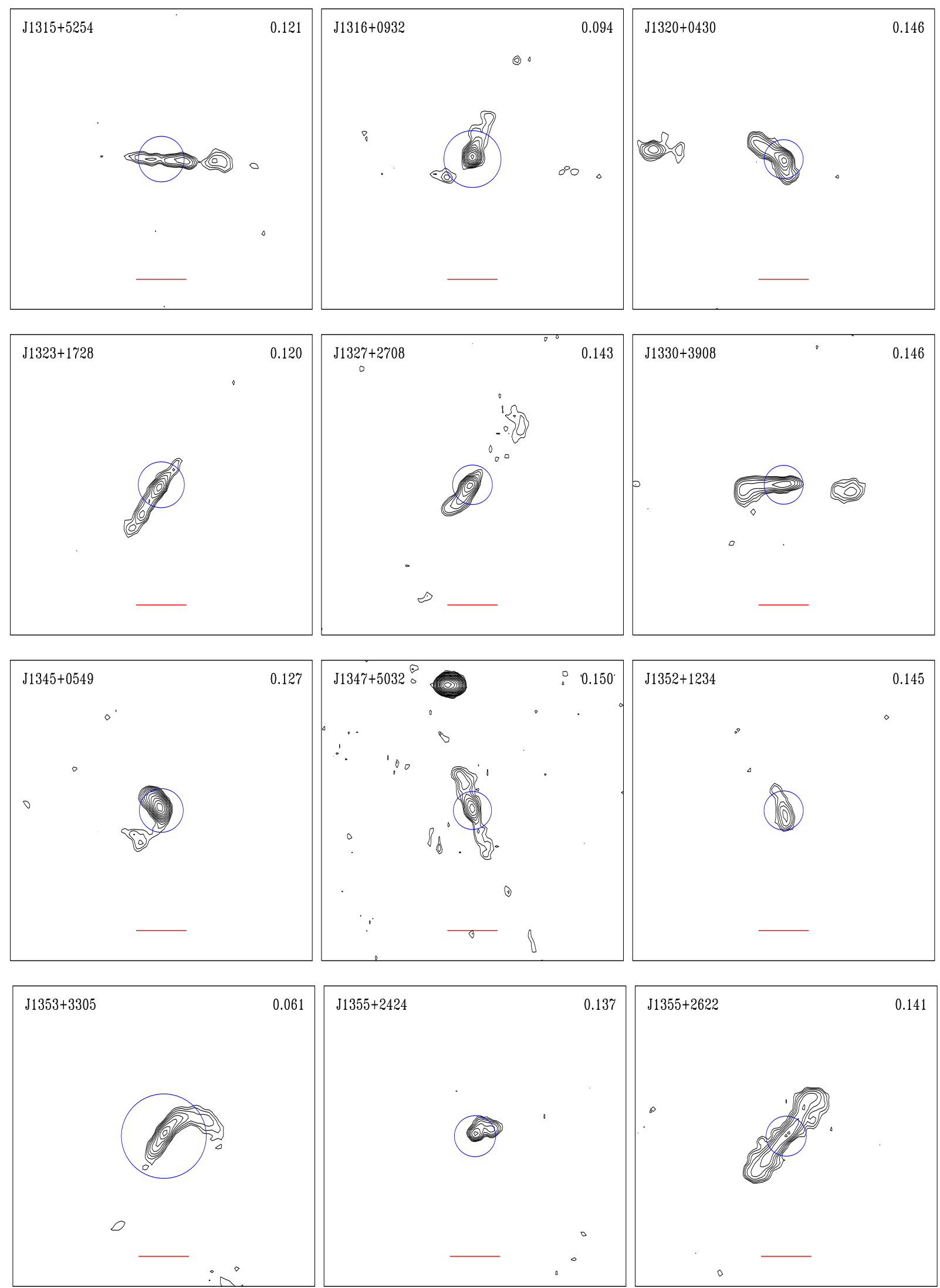

Fig. A.1. continued. 
A. Capetti et al.: FRICAT: A FIRST catalog of FR I radio galaxies
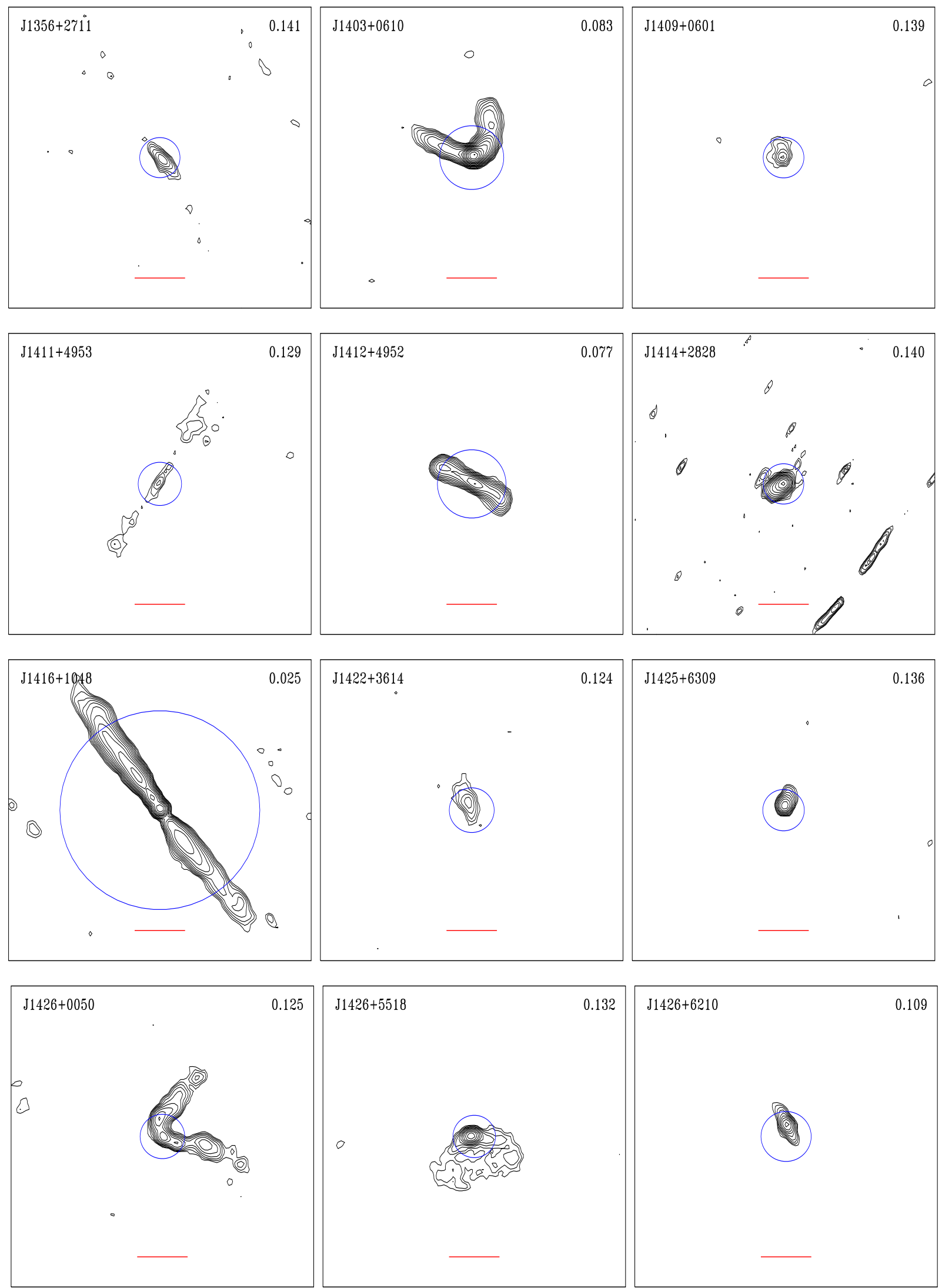

Fig. A.1. continued. 
A\&A 598, A49 (2017)
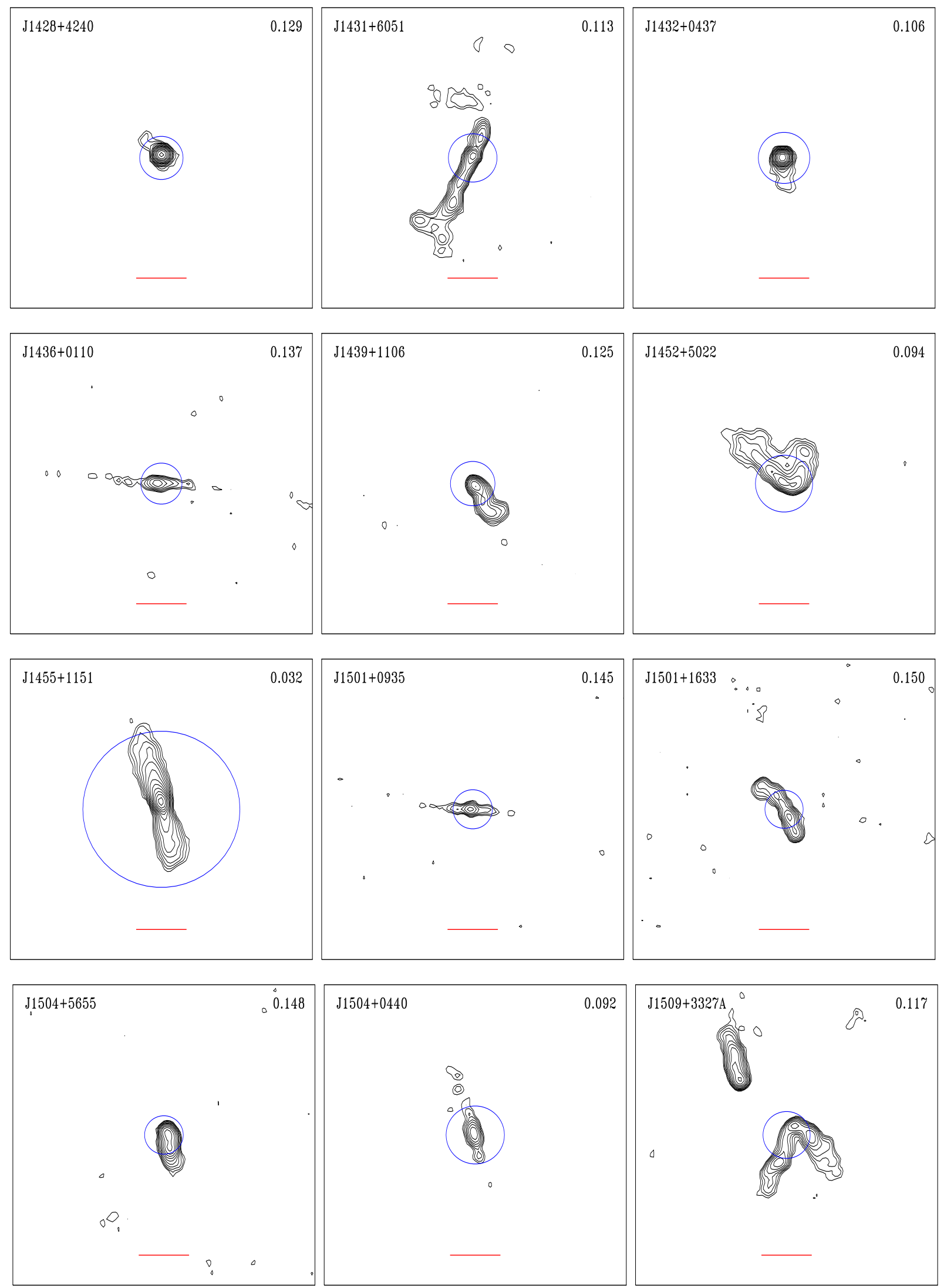

Fig. A.1. continued. 
A. Capetti et al.: FRICAT: A FIRST catalog of FR I radio galaxies
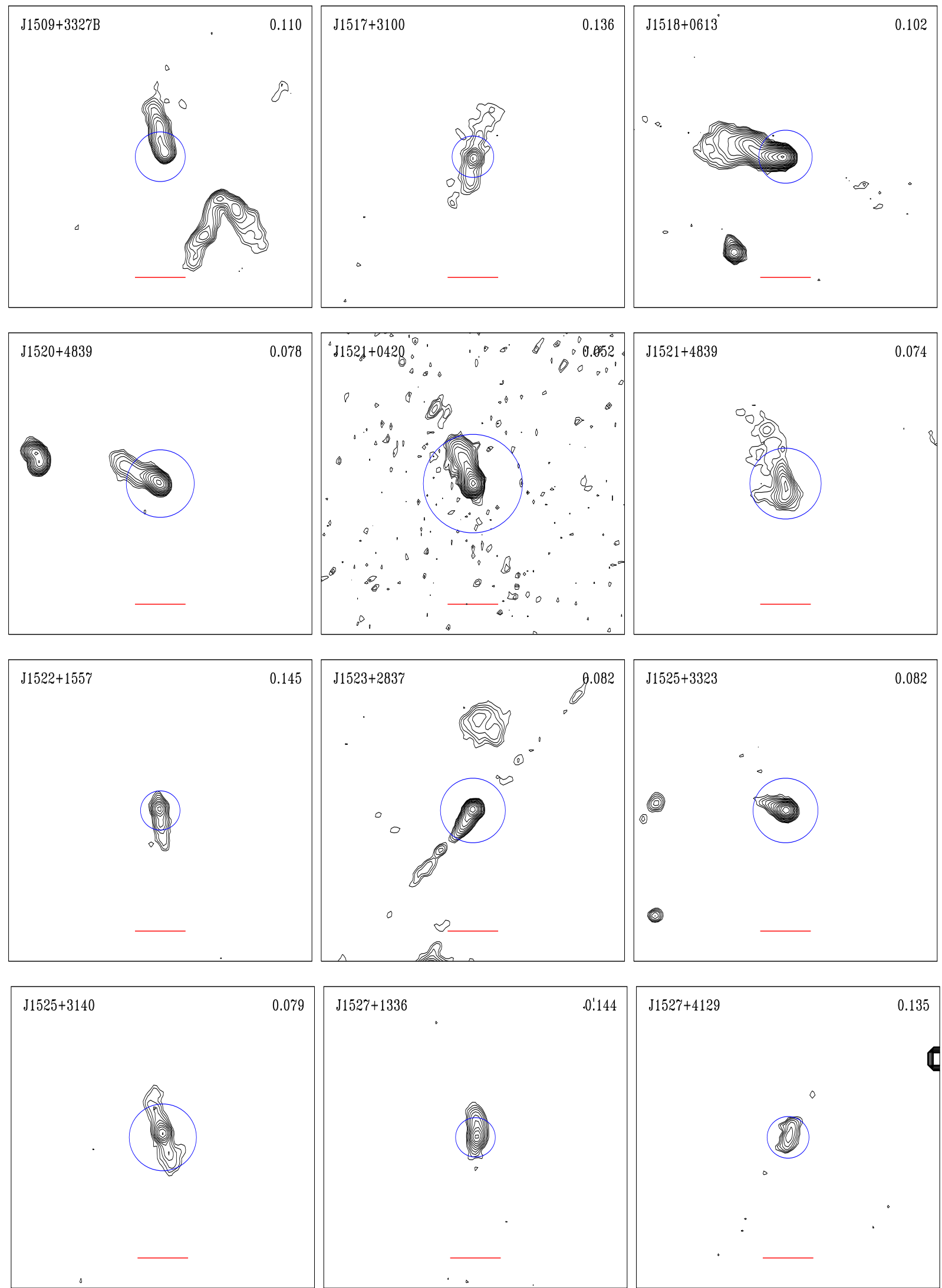

Fig. A.1. continued. 
A\&A 598, A49 (2017)
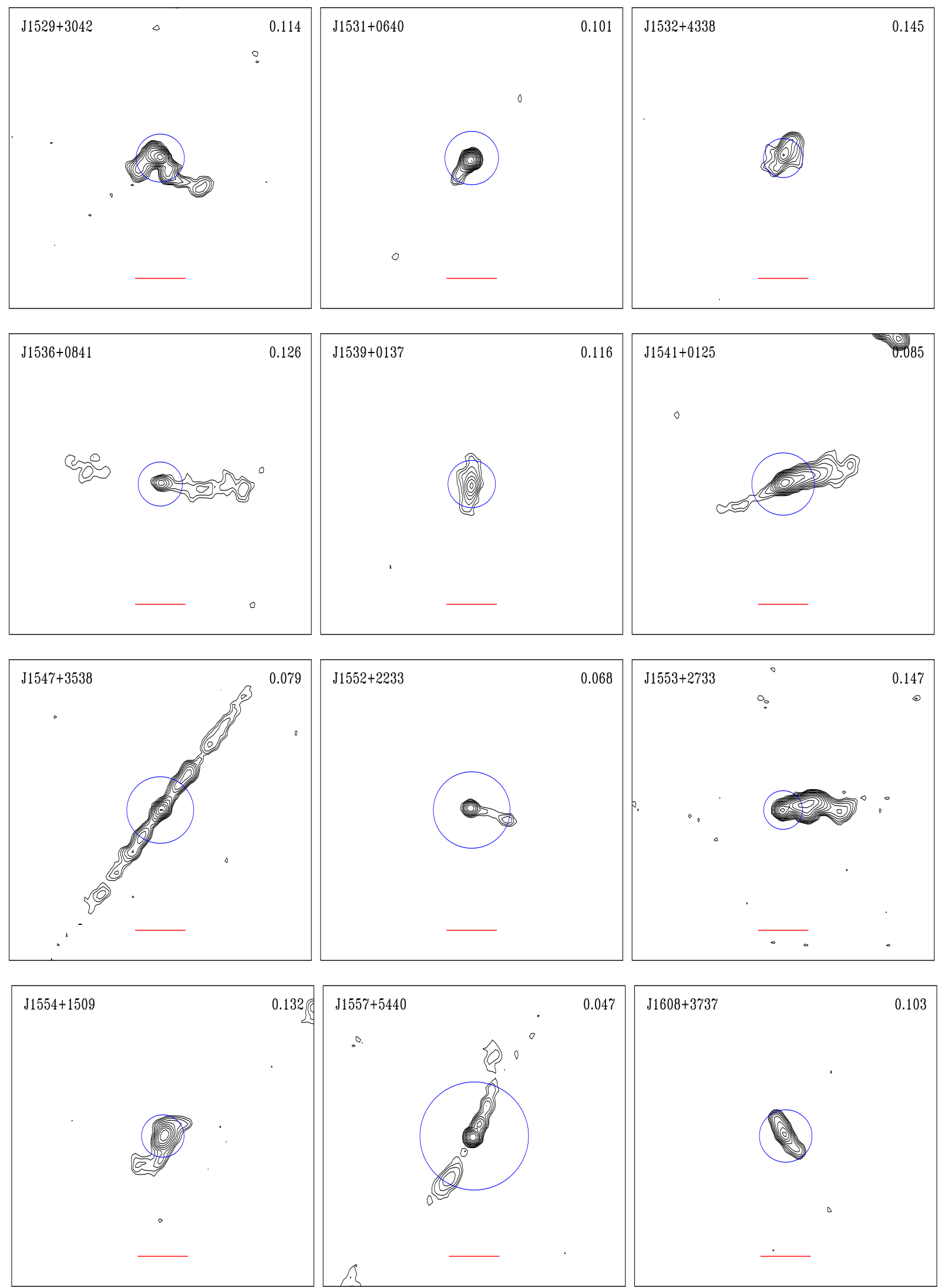

Fig. A.1. continued. 
A. Capetti et al.: FRICAT: A FIRST catalog of FR I radio galaxies
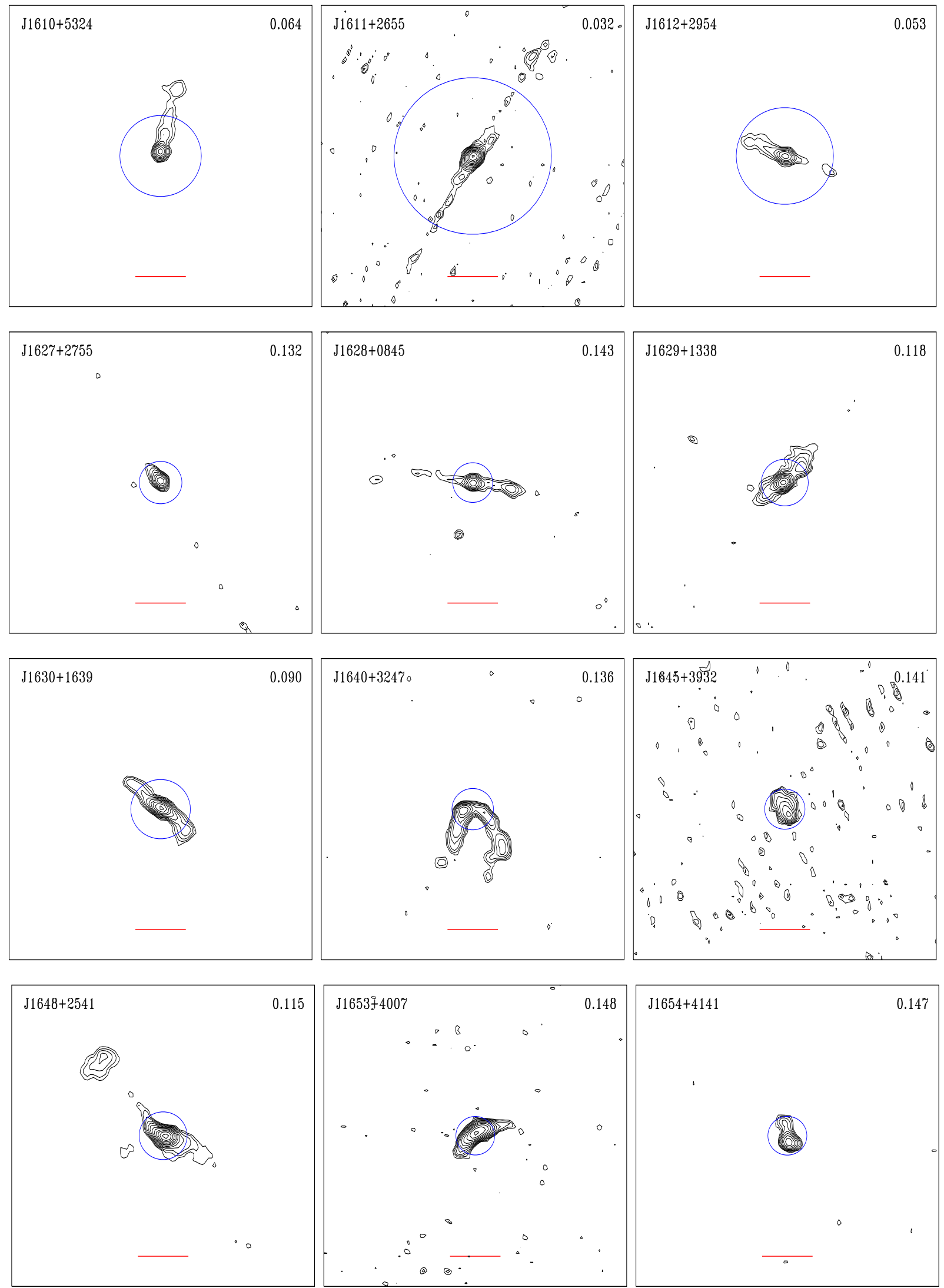

Fig. A.1. continued. 
A\&A 598, A49 (2017)
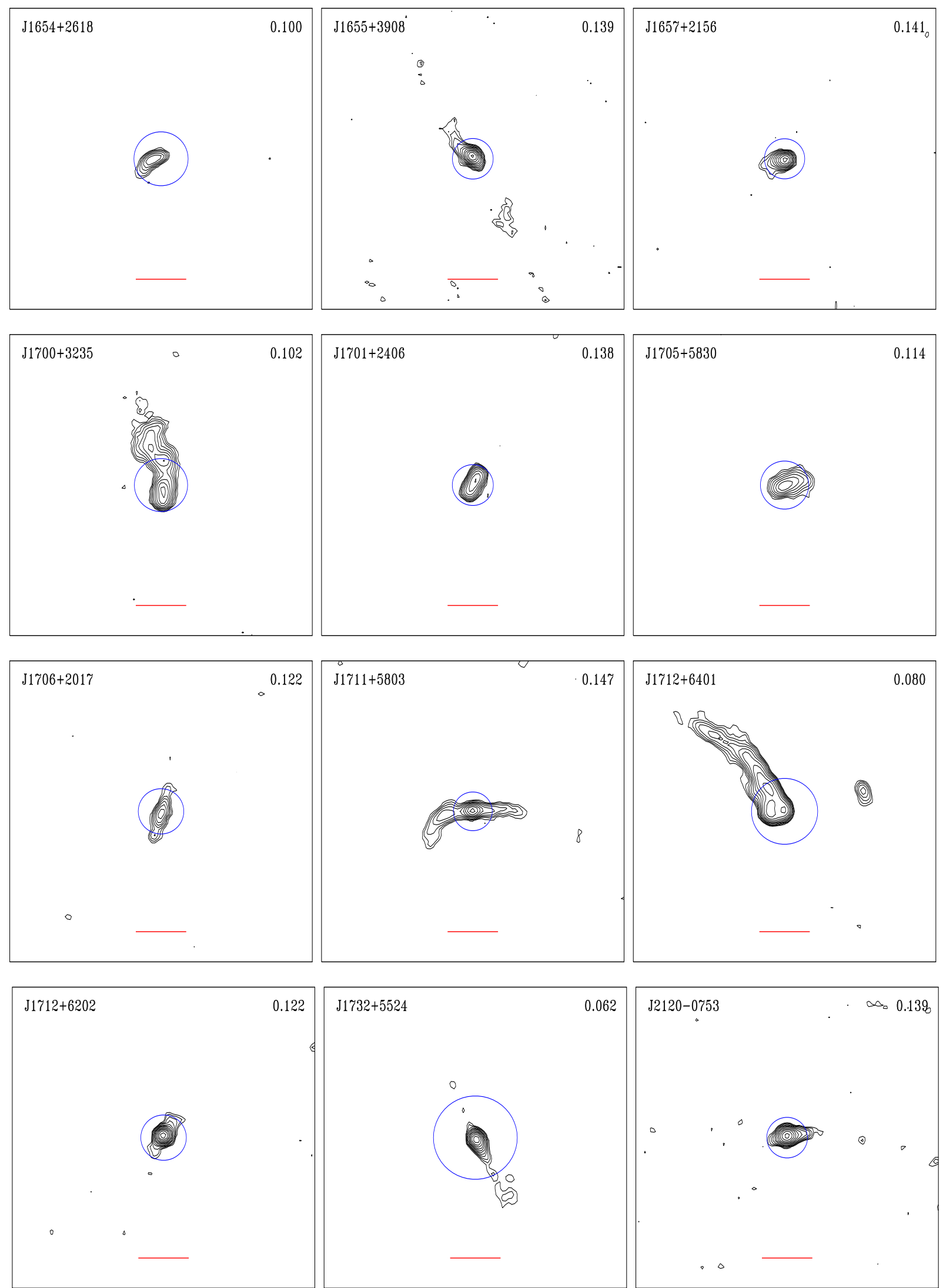

Fig. A.1. continued. 
A. Capetti et al.: FRICAT: A FIRST catalog of FR I radio galaxies

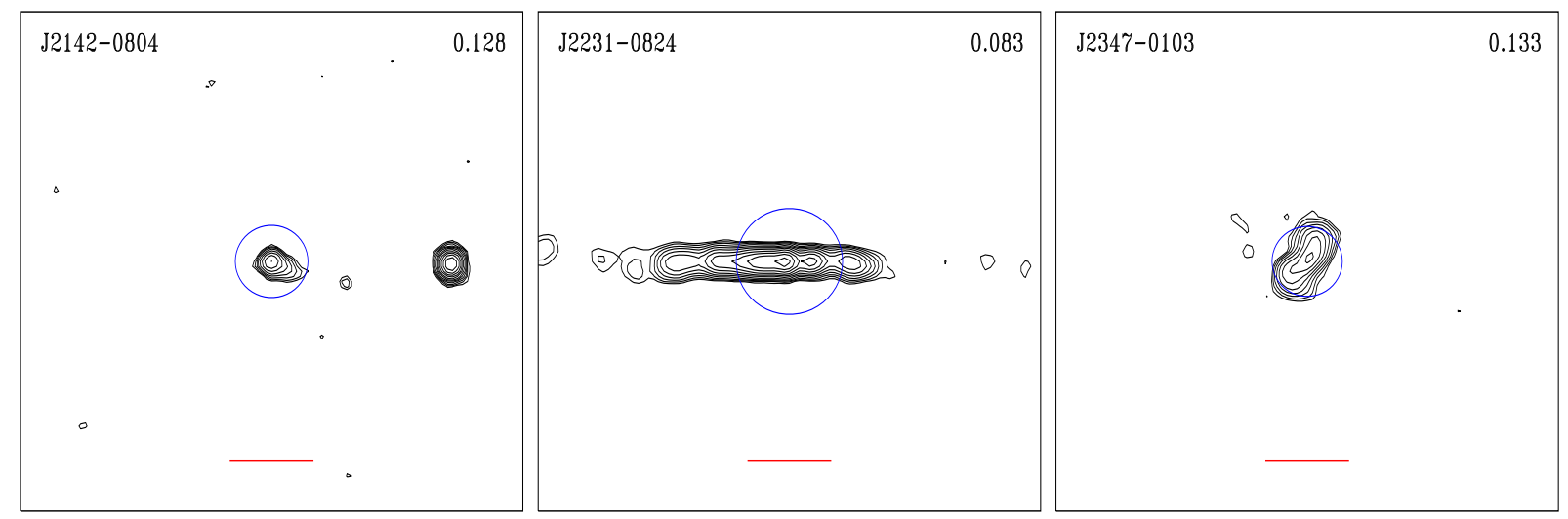

Fig. A.1. continued. 


\section{Appendix B: Additional tables}

Table B.1. Properties of the FRICAT sources.

\begin{tabular}{|c|c|c|c|c|c|c|c|c|c|c|}
\hline & $z$ & NVSS & [O III] & $m_{r}$ & Dn & $\sigma_{*}$ & $C_{r}$ & $v L_{r}$ & $\left.L_{[\mathrm{O}} \mathrm{III}\right]$ & $M_{\mathrm{BH}}$ \\
\hline SDSS J002900.98-011341.7 & 0.083 & 282.8 & 158.5 & 14.637 & 1.76 & 321 & 3.33 & 40.86 & 40.46 & 9.0 \\
\hline SDSS J003930.52-103218.6 & 0.129 & 23.7 & 1.9 & 15.996 & 1.97 & 238 & 3.14 & 40.19 & 38.94 & 8.4 \\
\hline SDSS J004148.22-091703.1 & 0.053 & 53.0 & 25.6 & 15.230 & 2.01 & 255 & 3.01 & 39.72 & 39.26 & 8.6 \\
\hline SDSS J004300.63-091346.3 & 0.076 & 148.4 & 40.7 & 15.437 & 1.96 & 244 & 3.32 & 40.50 & 39.79 & 8.5 \\
\hline SDSS J004530.46-004746.9 & 0.147 & 12.4 & 15.1 & 16.266 & 2.02 & 287 & 3.08 & 40.03 & 39.97 & 8.8 \\
\hline SDSS J011255.11-095040.6 & 0.124 & 81.7 & 20.8 & 16.026 & 1.84 & 220 & 2.99 & 40.69 & 39.95 & 8.3 \\
\hline SDSS J013327.25-082416.4 & 0.149 & 218.8 & 31.9 & 16.076 & 2.01 & 331 & 3.35 & 41.29 & 40.31 & 9.0 \\
\hline SDSS J013412.78-010729.4 & 0.079 & 119.6 & 39.6 & 15.152 & 2.03 & 262 & 3.55 & 40.44 & 39.81 & 8.6 \\
\hline SDSS J013503.43-005427.6 & 0.080 & 39.3 & 27.8 & 14.628 & 2.00 & 300 & 3.25 & 39.96 & 39.67 & 8.8 \\
\hline SDSS J014029.59+001825.8 & 0.148 & 12.3 & 8.2 & 16.642 & 1.95 & 278 & 3.34 & 40.03 & 39.71 & 8.7 \\
\hline SDSS J015253.79-001005.5 & 0.082 & 13.1 & 22.8 & 16.487 & 2.01 & 187 & 3.43 & 39.52 & 39.61 & 8.0 \\
\hline SDSS J025227.52-075605.4 & 0.078 & 99.7 & 10.4 & 15.348 & 1.95 & 237 & 3.00 & 40.35 & 39.22 & 8.4 \\
\hline SDSS J025437.99+005621.9 & 0.067 & 116.0 & 52.4 & 14.903 & 1.99 & 272 & 3.18 & 40.27 & 39.78 & 8.7 \\
\hline SDSS J073014.37+393200.4 & 0.142 & 11.3 & 5.3 & 16.716 & 2.07 & 223 & 3.08 & 39.96 & 39.48 & 8.3 \\
\hline SDSS J073505.25+415827.5 & 0.087 & 67.6 & 21.9 & 17.308 & 1.95 & 256 & 2.29 & 40.28 & 39.65 & 8.6 \\
\hline SDS & 0.111 & 43.0 & 4.7 & 16.038 & 2.03 & 231 & 2.84 & 40.30 & 39.19 & 8.4 \\
\hline SDSS J074125.85+480914.3 & 0.120 & 25.7 & 13.0 & 16.540 & 1.94 & 230 & 2.91 & 40.16 & 39.72 & 8.4 \\
\hline SDSS J074351.25+282128.0 & 0.106 & 20.2 & 15.5 & 16.206 & 1.94 & 252 & 3.34 & 39.94 & 39.68 & 8.5 \\
\hline SDSS J075309.91+355557.1 & 0.113 & 94.6 & 9.0 & 16.304 & 1.85 & 246 & 2.98 & 40.67 & 39.50 & 8.5 \\
\hline SDSS J075506.67+262115.9 & 0.123 & 46.0 & 3.1 & 16.483 & 1.94 & 265 & 3.03 & 40.43 & 39.12 & 8.6 \\
\hline+344030.8 & 0.083 & 45.0 & 5.5 & 15.641 & 2.03 & 226 & 3.13 & 40.06 & 39.00 & 8.3 \\
\hline SDSS J080326.62+303725.0 & 0.144 & & 1.0 & & & 301 & 2.97 & & & 8.8 \\
\hline SDSS J080923.10+211546.2 & 0.142 & 10.6 & 11.8 & 16.910 & 1.88 & 255 & 3.09 & 39.93 & 39.83 & 8.6 \\
\hline SDSS J081523.21+115715.1 & 0.100 & 40.0 & 3.6 & 15.468 & 1.85 & 251 & 3.27 & 40.18 & 39.00 & 8.5 \\
\hline SDSS J081604.40+112449.4 & 0.122 & 34.6 & 14.9 & 16.156 & 1.96 & 281 & 3.43 & 40.30 & 39.79 & 8.7 \\
\hline $27+425657.6$ & 0.127 & 121.0 & 11.3 & 16.628 & 1.98 & 240 & 3.09 & 40.88 & 39.70 & 8.4 \\
\hline $81849.74+040631.5$ & 0.095 & & 18.3 & & & 233 & & & & 8.4 \\
\hline SDSS J081932.66+200748.8 & 0.117 & 31.8 & 11.5 & 16.888 & 1.79 & 222 & 3.17 & 40.23 & 39.64 & 8.3 \\
\hline SDSS J082025.14+160123.2 & 0.148 & 44.2 & 19.3 & 17.505 & 2.00 & 225 & 2.92 & 40.59 & 40.08 & 8.3 \\
\hline SDSS J082028.09+485347.3 & & & 12.3 & & 2.01 & 295 & 2.72 & & 39.78 & 8.8 \\
\hline SDSS J082603.81+471910.3 & 0.128 & 25.7 & 2.3 & 16.288 & 1.98 & 231 & 3.49 & 40.22 & 39.02 & 8.4 \\
\hline SDSS J082729.73+530733.4 & 0.119 & 22.9 & 16.7 & & 1.88 & 184 & 2.93 & & & 8.0 \\
\hline SDSS J082733.62+160053.7 & 0.091 & 53.0 & 12.2 & 15.474 & 1.96 & 250 & 3.20 & 40.21 & 39.43 & 8.5 \\
\hline SDSS J082926.46+224436.3 & 0.087 & 148.8 & 9.3 & 15.623 & 2.00 & 223 & 3.19 & 40.62 & 39.27 & 8.3 \\
\hline SDSS J083138.83+223422.9 & 0.087 & 185.3 & 39.4 & & 1.89 & 302 & 3.32 & 40.72 & 39.90 & 8.8 \\
\hline SDSS J083159.69+303930.7 & 0.107 & 20.9 & 52.4 & 15.572 & 1.99 & 246 & 3.18 & 39.96 & 40.21 & 8.5 \\
\hline SDSS J083224.13+164949.1 & 0.102 & 20.2 & 23.6 & 15.896 & 1.92 & 304 & 3.37 & 39.90 & 39.83 & 8.9 \\
\hline SDSS J084140.57+254827.9 & 0.140 & 29.1 & 10.6 & 16.353 & 1.97 & 241 & 3.30 & 40.35 & 39.77 & 8.5 \\
\hline SDSS J084159.65+500551.7 & 0.141 & 7.6 & 3.8 & 16.677 & 2.06 & 259 & 3.15 & 39.78 & 39.33 & 8.6 \\
\hline SDSS J085321.54+331629.9 & 0.126 & 28.1 & 15.7 & 15.838 & 1.92 & 266 & 2.93 & 40.24 & 39.84 & 8.6 \\
\hline SDSS J085719.46+241142.6 & 0.133 & 11.8 & 10.9 & 16.694 & 2.06 & 220 & 3.20 & 39.92 & 39.73 & 8.3 \\
\hline SDSS J090018.16+074535.5 & 0.061 & 70.6 & 31.0 & 14.308 & 1.88 & 261 & 3.20 & 39.97 & 39.46 & 8.6 \\
\hline SDSS J090245.43+164710.4 & 0.130 & 15.2 & 7.9 & 16.049 & 2.02 & 243 & 3.16 & 40.00 & 39.57 & 8.5 \\
\hline SDSS J090543.54+401704.8 & 0.128 & 46.0 & 9.7 & 15.924 & 1.89 & 277 & 3.29 & 40.47 & 39.65 & 8.7 \\
\hline SDSS J091442.02+152155.7 & 0.140 & 28.3 & 7.0 & 16.839 & 1.86 & 235 & 3.32 & 40.35 & 39.60 & 8.4 \\
\hline SDSS J092049.04+403952.8 & 0.074 & 21.9 & 17.2 & 15.980 & 2.04 & 258 & 3.40 & 39.64 & 39.39 & 8.6 \\
\hline SDSS J092935.02+625659.3 & 0.121 & 50.9 & 19.4 & 15.785 & 1.90 & 281 & 3.11 & 40.46 & 39.89 & 8.7 \\
\hline SDSS J093058.74+034827.7 & 0.089 & 111.0 & 22.9 & 15.135 & 2.01 & 276 & 3.16 & 40.51 & 39.68 & 8.7 \\
\hline SDSS J093305.27+291015.1 & 0.132 & 53.6 & 12.3 & 15.743 & 1.97 & 254 & 3.34 & 40.56 & 39.78 & 8.5 \\
\hline
\end{tabular}

Notes. Column description: (1) source name; (2) redshift; (3) NVSS $1.4 \mathrm{GHz}$ flux density [mJy]; (4) [O III] flux [in $10^{-17} \mathrm{erg} \mathrm{cm}^{-2} \mathrm{~s}^{-1} \mathrm{units}$; (5) SDSS DR7 $r$ band $A B$ magnitude; (6) concentration index $C_{r}$; (7) Dn(4000) index; (8) stellar velocity dispersion [ $\mathrm{km} \mathrm{s}^{-1}$; ; (9) logarithm of the radio luminosity [ $\mathrm{erg} \mathrm{s}^{-1}$ ]; (10) logarithm of the [O III] line luminosity [erg s${ }^{-1}$ ]; (11) logarithm of the black hole mass [in solar units]. 
A. Capetti et al.: FRICAT: A FIRST catalog of FR I radio galaxies

Table B.1. continued.

\begin{tabular}{|c|c|c|c|c|c|c|c|c|c|c|}
\hline & $z$ & NVSS & [O III] & $m_{r}$ & Dn & $\sigma_{*}$ & $C_{r}$ & $v L_{r}$ & $\left.L_{[\mathrm{O}} \mathrm{III}\right]$ & $M_{\mathrm{BH}}$ \\
\hline SDSS J094202.04+105818.3 & 0.136 & 14.4 & 3.5 & 16.613 & 1.90 & 242 & 3.28 & 40.02 & 39.26 & 8.5 \\
\hline SDSS J094332.99+334158.3 & 0.131 & 23.9 & 2.8 & 15.974 & 1.91 & 276 & 3.24 & 40.21 & 39.14 & 8.7 \\
\hline SDSS J094614.50+581937.6 & 0.147 & 80.9 & 21.7 & 15.995 & 1.97 & 307 & 3.14 & 40.85 & 40.13 & 8.9 \\
\hline SDSS J095527.76+034516.8 & 0.091 & 47.0 & - & 16.615 & 1.81 & 190 & 3.10 & 40.16 & - & 8.0 \\
\hline SDSS J100451.83+543404.3 & 0.047 & 121.8 & 56.9 & 13.980 & 1.95 & 269 & 3.38 & 39.98 & 39.50 & 8.6 \\
\hline SDSS J100757.06+280147.9 & 0.148 & 27.3 & 11.5 & 15.864 & 1.99 & 309 & 3.26 & 40.38 & 39.86 & 8.9 \\
\hline SDSS J100804.13+502642.8 & 0.134 & 53.7 & 1.9 & 16.736 & 2.00 & 218 & 3.23 & 40.58 & 38.98 & 8.3 \\
\hline SDSS J101114.38+191425.7 & 0.149 & 31.5 & 11.8 & 16.531 & 1.89 & 269 & 3.38 & 40.45 & 39.87 & 8.6 \\
\hline SDSS J101545.46+311500.2 & 0.125 & 15.6 & 4.4 & 16.869 & 1.77 & 211 & 3.09 & 39.98 & 39.28 & 8.2 \\
\hline SDSS J101937.94+001955.7 & 0.096 & 43.0 & 31.7 & 15.251 & 1.96 & 275 & 3.16 & 40.17 & 39.89 & 8.7 \\
\hline SDSS J102008.61+174817.4 & 0.122 & 19.5 & 0.7 & 16.405 & 1.92 & 240 & 3.46 & 40.05 & 38.43 & 8.4 \\
\hline SDSS J102314.24+483122.0 & 0.148 & 31.1 & 17.0 & 16.331 & 1.98 & 296 & 3.24 & 40.44 & 40.03 & 8.8 \\
\hline SDSS J102603.83+390524.0 & 0.145 & 108.1 & 11.0 & 17.009 & 1.89 & 258 & 2.84 & 40.96 & 39.82 & 8.6 \\
\hline SDSS J102703.83+382013.0 & 0.123 & 41.0 & 9.0 & 15.916 & 1.87 & 209 & 3.17 & 40.38 & 39.58 & 8.2 \\
\hline SDSS J103036.15+355459.8 & 0.124 & 50.0 & 8.5 & 15.487 & 2.05 & 325 & 3.40 & 40.47 & 39.56 & 9.0 \\
\hline SDSS J103126.60+115250.5 & 0.144 & 25.2 & 12.7 & 16.291 & 1.98 & 259 & 3.15 & 40.32 & 39.87 & 8.6 \\
\hline SDSS J103258.88+564453.2 & 0.045 & 213.4 & 114.6 & 13.549 & 1.97 & 280 & 3.08 & 40.18 & 39.76 & 8.7 \\
\hline SDSS J103827.01+414852.9 & 0.125 & 43.0 & 14.7 & 16.366 & 1.82 & 193 & 3.23 & 40.42 & 39.80 & 8.1 \\
\hline SDSS J103930.43+394718.9 & 0.093 & 23.3 & 15.6 & 15.075 & 1.96 & 274 & 3.34 & 39.88 & 39.56 & 8.7 \\
\hline SDSS J104045.34+395448.5 & 0.134 & 35.8 & 2.5 & 16.888 & 1.91 & 235 & 2.98 & 40.40 & 39.11 & 8.4 \\
\hline SDSS J104049.99+561508.1 & 0.134 & 22.3 & 15.9 & 16.123 & 1.77 & 257 & 3.25 & 40.20 & 39.90 & 8.6 \\
\hline SDSS J104233.38+363946.5 & 0.142 & 53.1 & 2.0 & 16.347 & 1.82 & 243 & 2.95 & 40.63 & 39.06 & 8.5 \\
\hline SDSS J104855.28+311945.2 & 0.117 & 52.2 & 10.4 & 15.607 & 1.93 & 270 & 3.08 & 40.44 & 39.59 & 8.7 \\
\hline SDSS J104907.26+551314.9 & 0.126 & 24.0 & 23.8 & 15.440 & 1.89 & 448 & 3.30 & 40.17 & 40.02 & 9.5 \\
\hline SDSS J104921.13-004005.0 & 0.039 & 250.0 & 70.2 & 13.544 & 1.90 & 226 & 2.83 & 40.11 & 39.42 & 8.3 \\
\hline SDSS J105147.39+552308.3 & 0.074 & 522.0 & 48.5 & 14.624 & 2.08 & 320 & 3.54 & 41.02 & 39.84 & 9.0 \\
\hline SDSS J105259.97+430255.0 & 0.148 & 52.0 & 23.6 & 16.414 & 1.87 & 225 & 3.32 & 40.66 & 40.17 & 8.3 \\
\hline SDSS J105344.12+492955.9 & 0.140 & 64.3 & 33.4 & 16.012 & 1.30 & 262 & 3.49 & 40.70 & 40.27 & 8.6 \\
\hline SDSS J105348.93+402345.9 & 0.128 & 100.1 & 1.2 & 15.592 & 1.97 & 283 & 3.22 & 40.81 & 38.72 & 8.7 \\
\hline SDSS J105544.98+452401.4 & 0.064 & 133.5 & 6.8 & 14.706 & 2.02 & 213 & 3.34 & 40.29 & 38.85 & 8.2 \\
\hline SDSS J105702.79+564503.1 & 0.136 & 15.4 & 4.1 & 16.207 & 2.02 & 304 & 3.39 & 40.05 & 39.33 & 8.9 \\
\hline SDSS J105847.67+164526.0 & 0.116 & 49.4 & 16.4 & 16.261 & 1.88 & 226 & 3.35 & 40.41 & 39.79 & 8.3 \\
\hline SDSS J110535.78+091956.3 & 0.127 & 22.3 & 6.1 & 16.110 & 2.00 & 251 & 3.23 & 40.15 & 39.44 & 8.5 \\
\hline SDSS J111020.07+204657.5 & 0.135 & 12.9 & 2.9 & 16.509 & 1.97 & 275 & 3.38 & 39.97 & 39.18 & 8.7 \\
\hline SDSS J111037.33+541135.7 & 0.141 & 23.5 & 15.7 & 16.449 & 1.99 & 206 & 3.08 & 40.27 & 39.95 & 8.2 \\
\hline SDSS J111211.37+304352.3 & 0.106 & 43.5 & 24.5 & 15.990 & 1.95 & 244 & 3.29 & 40.27 & 39.87 & 8.5 \\
\hline SDSS J111337.13+234846.5 & 0.140 & 8.4 & 17.4 & 16.842 & 1.96 & 249 & 3.33 & 39.82 & 39.99 & 8.5 \\
\hline SDSS J111911.13+081539.8 & 0.076 & 80.0 & 25.6 & 15.054 & 2.05 & 257 & 3.16 & 40.22 & 39.58 & 8.6 \\
\hline SDSS J112055.83+173854.0 & 0.085 & 21.5 & 9.4 & 15.623 & 1.97 & 205 & 2.85 & 39.75 & 39.25 & 8.2 \\
\hline SDSS J112352.34+443735.6 & 0.139 & 22.9 & 10.1 & 17.434 & 1.90 & 273 & 3.85 & 40.24 & 39.74 & 8.7 \\
\hline SDSS J112403.19+475814.9 & 0.140 & 28.2 & 3.0 & 16.675 & 1.97 & 266 & 3.22 & 40.35 & 39.22 & 8.6 \\
\hline SDSS J112457.40+171744.7 & 0.142 & 9.5 & 5.0 & 16.147 & 2.00 & 285 & 3.03 & 39.88 & 39.46 & 8.8 \\
\hline SDSS J112603.59+545329.1 & 0.149 & 16.0 & 12.4 & 16.871 & 1.94 & 258 & 3.46 & 40.16 & 39.90 & 8.6 \\
\hline SDSS J113012.79+235822.1 & 0.140 & 46.5 & 19.8 & 16.474 & 1.80 & 265 & 3.60 & 40.56 & 40.05 & 8.6 \\
\hline SDSS J113359.23+490343.4 & 0.032 & 732.0 & 84.7 & 13.126 & 1.90 & 264 & 3.30 & 40.40 & 39.32 & 8.6 \\
\hline SDSS J114210.72+552729.6 & 0.133 & 24.9 & 8.8 & 16.099 & 2.01 & 218 & 3.05 & 40.24 & 39.64 & 8.3 \\
\hline SDSS J114212.11+101159.0 & 0.103 & 46.6 & 9.1 & 16.086 & 1.89 & 243 & 3.10 & 40.28 & 39.42 & 8.5 \\
\hline SDSS J114345.53+192333.4 & 0.094 & 37.8 & 17.0 & 15.411 & 2.00 & 237 & 3.17 & 40.10 & 39.61 & 8.4 \\
\hline SDSS J115109.39+435918.6 & 0.071 & 75.0 & 37.5 & 14.982 & 1.92 & 216 & 3.17 & 40.13 & 39.69 & 8.3 \\
\hline SDSS J115323.89+305904.8 & 0.136 & 43.2 & 8.9 & 16.911 & 1.97 & 191 & 3.30 & 40.50 & 39.67 & 8.1 \\
\hline SDSS J115508.97+232623.4 & 0.144 & 26.4 & 12.6 & 17.279 & 2.00 & 245 & 2.98 & 40.34 & 39.87 & 8.5 \\
\hline SDSS J115729.60+292308.1 & 0.140 & 16.6 & 9.3 & 17.195 & 2.01 & 224 & 3.32 & 40.11 & 39.71 & 8.3 \\
\hline SDSS J115816.37+340605.9 & 0.131 & 19.8 & 26.3 & 16.311 & 1.85 & 221 & 3.16 & 40.13 & 40.11 & 8.3 \\
\hline SDSS J115936.05+233947.5 & 0.142 & 31.8 & 5.0 & 16.510 & 1.92 & 282 & 3.51 & 40.41 & 39.45 & 8.7 \\
\hline
\end{tabular}


Table B.1. continued.

\begin{tabular}{|c|c|c|c|c|c|c|c|c|c|c|}
\hline & $z$ & NVSS & [O III] & $m_{r}$ & $\mathrm{Dn}$ & $\sigma_{*}$ & $C_{r}$ & $v L_{r}$ & $\left.L_{[\mathrm{O}} \mathrm{III}\right]$ & $M_{\mathrm{BH}}$ \\
\hline SDSS J120021.93-020152.7 & 0.146 & 40.4 & 24.8 & 16.336 & 2.04 & 248 & 3.12 & 40.54 & 40.18 & 8.5 \\
\hline SDSS J120401.47+201356.3 & 0.024 & 402.1 & 111.6 & 12.997 & 1.81 & 270 & 3.25 & 39.91 & 39.21 & 8.7 \\
\hline SDSS J120425.29+034510.6 & 0.149 & 54.7 & 4.2 & 16.608 & 1.95 & 248 & 3.14 & 40.69 & 39.43 & 8.5 \\
\hline SDSS J120522.29+050941.4 & 0.136 & 105.0 & 0.6 & 16.002 & 2.02 & 335 & 3.47 & 40.88 & 38.49 & 9.0 \\
\hline SDSS J120943.62-020459.6 & 0.100 & 32.3 & 23.3 & 15.922 & 1.98 & 253 & 3.34 & 40.09 & 39.80 & 8.5 \\
\hline SDSS J121110.99+060744.1 & 0.139 & 50.9 & 12.8 & 16.154 & 1.94 & 221 & 3.12 & 40.59 & 39.84 & 8.3 \\
\hline SDSS J121114.07+060833.9 & 0.138 & 78.5 & 11.1 & 16.143 & 1.96 & 238 & 3.18 & 40.78 & 39.78 & 8.4 \\
\hline SDSS J121121.12+141439.2 & 0.064 & 62.0 & 56.4 & 14.783 & 2.03 & 233 & 3.13 & 39.96 & 39.77 & 8.4 \\
\hline SDSS J121332.93+072516.9 & 0.137 & 17.2 & 10.9 & 15.739 & 1.92 & 242 & 3.00 & 40.11 & 39.76 & 8.5 \\
\hline SDSS J121519.19+472142.4 & 0.146 & 31.1 & 2.9 & 17.108 & 0.00 & 193 & 3.09 & 40.42 & 39.25 & 8.1 \\
\hline SDSS J121534.18+135635.0 & 0.093 & 30.9 & 21.1 & 15.362 & 2.04 & 303 & 3.15 & 40.00 & 39.69 & 8.9 \\
\hline SDSS J121543.82+170917.6 & 0.095 & 460.0 & 40.0 & 14.538 & 1.99 & 312 & 2.86 & 41.19 & 39.99 & 8.9 \\
\hline SDSS J121619.95+155417.7 & 0.093 & 77.2 & 19.0 & 15.228 & 1.81 & 210 & 3.11 & 40.40 & 39.64 & 8.2 \\
\hline SDSS J121640.12+034231.5 & 0.080 & 207.0 & 24.0 & 15.677 & 1.99 & 243 & 3.22 & 40.69 & 39.61 & 8.5 \\
\hline SDSS J122156.16+020450.8 & 0.126 & 119.5 & 9.5 & 16.885 & 1.92 & 232 & 3.40 & 40.87 & 39.62 & 8.4 \\
\hline SDSS J122532.09+192615.2 & 0.129 & 25.6 & 23.9 & 17.363 & 1.82 & 192 & 3.05 & 40.22 & 40.05 & 8.1 \\
\hline SDSS J122622.49+640622.0 & 0.110 & 81.0 & 43.6 & 15.516 & 1.82 & 257 & 3.09 & 40.58 & 40.16 & 8.6 \\
\hline SDSS J122640.83+430509.2 & 0.074 & 33.5 & 18.4 & 14.836 & 1.98 & 272 & 3.15 & 39.83 & 39.42 & 8.7 \\
\hline SDSS J123128.93+491537.0 & 0.111 & 52.1 & 5.1 & 16.192 & 1.85 & 217 & 3.06 & 40.39 & 39.24 & 8.3 \\
\hline SDSS J124135.94+162033.6 & 0.070 & 165.0 & 77.2 & 15.040 & 1.95 & 305 & 3.31 & 40.47 & 39.99 & 8.9 \\
\hline SDSS J124207.38+502146.6 & 0.148 & 102.0 & 14.3 & 16.365 & 1.97 & 237 & 3.33 & 40.95 & 39.95 & 8.4 \\
\hline SDSS J124622.48+075327.9 & 0.111 & 19.8 & 23.4 & 15.774 & 1.95 & 275 & 3.25 & 39.97 & 39.89 & 8.7 \\
\hline SDSS J124647.52+545315.0 & 0.085 & 38.5 & 32.6 & 14.974 & 2.01 & 268 & 3.09 & 40.01 & 39.79 & 8.6 \\
\hline SDSS J125434.93-023412.4 & 0.116 & 122.6 & 2.0 & 15.959 & 1.93 & 260 & 3.30 & 40.80 & 38.86 & 8.6 \\
\hline $32+575149.7$ & 0.149 & 15 & 5.5 & 17.347 & 1.71 & 211 & 3.17 & 40.14 & 39.55 & 8.2 \\
\hline SDSS J130203.58-005012.3 & 0.085 & 73.7 & 30.2 & 14.964 & 2.10 & 243 & 3.27 & 40.29 & 39.76 & 8.5 \\
\hline SDSS J130248.70+475510.6 & 0.141 & 47.9 & 20.0 & 15.563 & 1.95 & 301 & 3.06 & 40.58 & 40.05 & 8.8 \\
\hline SDSS J130619.24+111339.7 & 0.086 & 321.0 & 44.5 & 14.926 & 1.72 & 267 & 3.04 & 40.94 & 39.94 & 8.6 \\
\hline SDSS J131053.44-022841.5 & 0.143 & 53.0 & 12.7 & 16.792 & 2.04 & 267 & 2.77 & 40.64 & 39.87 & 8.6 \\
\hline SDSS J131531.07+525437.3 & 0.121 & 37.1 & 0.5 & 16.470 & 2.01 & 247 & 3.31 & 40.33 & 38.30 & 8.5 \\
\hline SDSS J131613.54+093236.7 & 0.094 & 42.0 & 23.6 & 15.558 & 1.95 & 293 & 3.43 & 40.14 & 39.74 & 8.8 \\
\hline SDSS J132017.54+043037.4 & 0.146 & 30.5 & 4.4 & 15.935 & 1.81 & 229 & 2.59 & 40.42 & 39.42 & 8.4 \\
\hline SDSS J132302.49+172832.9 & 0.120 & 38.7 & 13.9 & 16.128 & 1.92 & 242 & 3.36 & 40.34 & 39.75 & 8.5 \\
\hline SDSS J132736.13+270816.8 & 0.143 & 34.2 & 11.2 & 16.081 & 2.03 & 267 & 3.35 & 40.45 & 39.82 & 8.6 \\
\hline SDSS J133038.01+390815.4 & 0.146 & 47.5 & 14.3 & 16.858 & 1.93 & 236 & 3.22 & 40.61 & 39.94 & 8.4 \\
\hline SDSS J13 & 0.127 & 54 & 15.8 & 98 & 1.97 & 240 & 3.21 & 40.53 & 39.85 & 8.4 \\
\hline SDSS J134745.19+503203.5 & 0.150 & 18.2 & 1.3 & 16.204 & 2.06 & 244 & 3.35 & 40.21 & 38.91 & 8.5 \\
\hline SDSS J135214.56+123401.7 & 0.145 & 11.0 & - & 16.729 & 2.02 & 239 & 3.44 & 39.96 & - & 8.4 \\
\hline SDSS J135302.04+330528.5 & 0.061 & 80.0 & 22.1 & 14.703 & 1.93 & 208 & 3.24 & 40.03 & 39.32 & 8.2 \\
\hline SDSS J135511.34+242415.6 & 0.137 & 16.1 & 4.1 & 16.713 & 1.90 & 270 & 3.08 & 40.08 & 39.34 & 8.7 \\
\hline SDSS J135553.63+262217.9 & 0.141 & 69.8 & 6.9 & 15.904 & 1.94 & 294 & 3.17 & 40.74 & 39.59 & 8.8 \\
\hline SDSS J135655.28+271120.2 & 0.141 & 16.3 & 13.5 & 16.061 & 1.93 & 253 & 3.06 & 40.11 & 39.88 & 8.5 \\
\hline SDSS J140313.28+061008.2 & 0.083 & 256.6 & 76.9 & 15.097 & 1.87 & 340 & 3.23 & 40.81 & 40.14 & 9.1 \\
\hline SDSS J140916.74+060139.4 & 0.139 & 10.8 & 25.3 & 16.844 & 1.91 & 218 & 3.37 & 39.92 & 40.14 & 8.3 \\
\hline SDSS J141138.22+495304.0 & 0.129 & 27.5 & 16.5 & 16.478 & 2.12 & 230 & 3.10 & 40.25 & 39.88 & 8.4 \\
\hline SDSS J141243.83+495206.5 & 0.077 & 101.4 & 24.0 & 14.643 & 2.04 & 303 & 3.23 & 40.35 & 39.58 & 8.9 \\
\hline SDSS J141427.10+282830.5 & 0.140 & 77.1 & 9.4 & 16.420 & 1.72 & 263 & 3.30 & 40.78 & 39.72 & 8.6 \\
\hline SDSS J141652.94+104826.7 & 0.025 & 4581.1 & 228.7 & 12.057 & 1.97 & 341 & 3.06 & 40.98 & 39.53 & 9.1 \\
\hline SDSS J142206.79+361434.8 & 0.124 & 13.7 & 17.5 & 16.742 & 1.73 & 226 & 2.75 & 39.91 & 39.87 & 8.3 \\
\hline SDSS J142521.22+630921.3 & 0.136 & 19.8 & 7.7 & 15.833 & 1.89 & 273 & 3.22 & 40.16 & 39.60 & 8.7 \\
\hline SDSS J142616.34+005015.3 & 0.125 & 88.7 & 10.1 & 15.878 & 1.97 & 254 & 2.98 & 40.73 & 39.64 & 8.6 \\
\hline SDSS J142623.76+551804.9 & 0.132 & 52.8 & 8.5 & 16.254 & 1.96 & 205 & 3.30 & 40.56 & 39.62 & 8.2 \\
\hline SDSS J142649.23+621005.9 & 0.109 & 25.9 & 28.5 & 15.539 & 1.94 & 306 & 3.40 & 40.07 & 39.97 & 8.9 \\
\hline SDSS J142832.60+424021.0 & 0.129 & 56.4 & 48.0 & 16.182 & 1.18 & 251 & 3.23 & 40.57 & 40.35 & 8.5 \\
\hline
\end{tabular}


A. Capetti et al.: FRICAT: A FIRST catalog of FR I radio galaxies

Table B.1. continued.

\begin{tabular}{|c|c|c|c|c|c|c|c|c|c|c|}
\hline & $z$ & NVSS & [O III] & $m_{r}$ & Dn & $\sigma_{*}$ & $C_{r}$ & $v L_{r}$ & $\left.L_{[\mathrm{O}} \mathrm{III}\right]$ & $M_{\mathrm{BH}}$ \\
\hline SDSS J143147.54+605109.4 & 0.113 & 163.0 & 27.7 & 15.726 & 1.94 & 251 & 3.19 & 40.90 & 39.99 & 8.5 \\
\hline SDSS J143257.81+043715.1 & 0.106 & 68.6 & 21.8 & 15.767 & 1.99 & 302 & 3.14 & 40.46 & 39.82 & 8.9 \\
\hline SDSS J143638.56+011058.8 & 0.137 & 19.2 & 15.9 & 15.944 & 2.02 & 326 & 2.98 & 40.16 & 39.93 & 9.0 \\
\hline SDSS J143928.78+110613.8 & 0.125 & 39.8 & 12.7 & 16.068 & 1.67 & 247 & 2.86 & 40.39 & 39.74 & 8.5 \\
\hline SDSS J145215.46+502225.1 & 0.094 & 133.8 & 13.9 & 15.124 & 1.99 & 246 & 3.14 & 40.65 & 39.52 & 8.5 \\
\hline SDSS J145555.27+115141.4 & 0.032 & 382.0 & 142.0 & 13.224 & 1.98 & 287 & 3.30 & 40.12 & 39.54 & 8.8 \\
\hline SDSS J150111.50+093547.9 & 0.145 & 15.2 & 15.3 & 16.549 & 1.98 & 246 & 3.09 & 40.10 & 39.96 & 8.5 \\
\hline SDSS J150148.14+163345.6 & 0.150 & 37.2 & 3.9 & 16.488 & 1.98 & 225 & 3.36 & 40.53 & 39.40 & 8.3 \\
\hline SDSS J150408.01+565545.4 & 0.148 & 57.3 & 12.4 & 16.708 & 1.98 & 276 & 3.30 & 40.70 & 39.89 & 8.7 \\
\hline SDSS J150450.51+044054.8 & 0.092 & 53.6 & 7.7 & 15.848 & 1.89 & 227 & 2.99 & 40.23 & 39.24 & 8.3 \\
\hline SDSS J150957.37+332715.0 & 0.117 & 66.0 & 11.7 & 15.956 & 1.97 & 266 & 3.24 & 40.54 & 39.65 & 8.6 \\
\hline SDSS J150959.74+332746.1 & 0.110 & 66.0 & 25.0 & 16.560 & 1.90 & 232 & 3.10 & 40.49 & 39.92 & 8.4 \\
\hline SDSS J151744.96+310015.8 & 0.136 & 49.0 & 7.1 & 16.224 & 1.94 & 291 & 3.21 & 40.55 & 39.57 & 8.8 \\
\hline SDSS J151845.72+061356.1 & 0.102 & 487.0 & 26.0 & 15.589 & 1.31 & 297 & 3.63 & 41.28 & 39.86 & 8.8 \\
\hline SDSS J152045.04+483922.9 & 0.078 & 80.6 & 19.8 & 15.917 & 2.12 & 257 & 3.04 & 40.26 & 39.50 & 8.6 \\
\hline SDSS J152122.54+042030.1 & 0.052 & 452.0 & 51.4 & 13.936 & 1.85 & 280 & 2.93 & 40.64 & 39.55 & 8.7 \\
\hline SDSS J152126.99+483943.2 & 0.074 & 63.3 & 17.4 & 15.628 & 2.10 & 256 & 3.14 & 40.10 & 39.40 & 8.6 \\
\hline SDSS J152235.19+155707.6 & 0.145 & 25.2 & 15.2 & 16.575 & 2.11 & 298 & 3.28 & 40.33 & 39.96 & 8.8 \\
\hline SDSS J152326.91+283732.5 & 0.082 & 733.0 & 48.9 & 15.021 & 1.93 & 234 & 3.16 & 41.26 & 39.94 & 8.4 \\
\hline SDSS J152500.83+332359.8 & 0.082 & 75.5 & 52.6 & 15.232 & 1.84 & 243 & 3.21 & 40.27 & 39.96 & 8.5 \\
\hline SDSS J152522.33+314037.1 & 0.079 & 51.4 & 33.7 & 15.541 & 2.01 & 250 & 3.35 & 40.07 & 39.74 & 8.5 \\
\hline SDSS J152715.31+133650.9 & 0.144 & 47.2 & 25.9 & 16.172 & 2.00 & 291 & 2.88 & 40.59 & 40.19 & 8.8 \\
\hline SDSS J152737.36+412947.1 & 0.135 & 14.3 & - & 16.562 & 2.00 & 222 & 3.07 & 40.02 & - & 8.3 \\
\hline SDSS J152945.60+304235.6 & 0.114 & 88.0 & 27.8 & 15.252 & 2.05 & 356 & 3.55 & 40.64 & 40.00 & 9.1 \\
\hline SDSS J153138.76+064045.5 & 0.101 & 40.1 & 47.2 & 14.959 & 1.83 & 312 & 3.25 & 40.19 & 40.12 & 8.9 \\
\hline SDSS J153215.31+433844.5 & 0.145 & 26.8 & 15.9 & 16.593 & 1.96 & 250 & 3.17 & 40.35 & 39.98 & 8.5 \\
\hline SDSS J153621.11+084112.1 & 0.126 & 68.1 & 18.6 & 16.255 & 2.00 & 266 & 3.31 & 40.63 & 39.92 & 8.6 \\
\hline SDSS J153932.09+013710.5 & 0.116 & 20.1 & 26.6 & 16.279 & 1.99 & 278 & 2.93 & 40.02 & 39.99 & 8.7 \\
\hline SDSS J154155.16+012517.4 & 0.085 & 113.0 & 24.5 & 15.291 & 1.96 & 276 & 3.25 & 40.48 & 39.67 & 8.7 \\
\hline SDSS J154709.22+353846.1 & 0.079 & 213.9 & 36.2 & 14.574 & 2.06 & 355 & 3.01 & 40.70 & 39.78 & 9.1 \\
\hline SDSS J155222.36+223311.9 & 0.068 & 44.8 & 40.0 & 14.802 & 2.04 & 267 & 3.44 & 39.88 & 39.68 & 8.6 \\
\hline SDSS J155311.93+273320.6 & 0.147 & 115.0 & 9.0 & 15.829 & 1.98 & 271 & 2.89 & 41.00 & 39.75 & 8.7 \\
\hline SDSS J155401.99+150946.8 & 0.132 & 50.5 & 19.7 & 16.590 & 1.99 & 240 & 3.21 & 40.54 & 39.98 & 8.5 \\
\hline SDSS J155721.38+544015.9 & 0.047 & 90.0 & 91.8 & 13.930 & 1.79 & 289 & 3.44 & 39.84 & 39.71 & 8.8 \\
\hline SDSS J160816.32+373743.1 & 0.103 & 40.4 & 13.3 & 16.018 & 1.99 & 248 & 3.30 & 40.21 & 39.59 & 8.5 \\
\hline SDSS J161037.77+532421.0 & 0.064 & 61.2 & 48.6 & 14.847 & 2.02 & 287 & 3.43 & 39.95 & 39.71 & 8.8 \\
\hline SDSS J161114.11+265524.2 & 0.032 & 102.8 & 111.1 & 13.437 & 1.97 & 263 & 3.42 & 39.56 & 39.44 & 8.6 \\
\hline SDSS J161242.69+295404.7 & 0.053 & 36.1 & 5.6 & 14.666 & 1.98 & 215 & 3.29 & 39.56 & 38.61 & 8.3 \\
\hline SDSS J162700.42+275547.7 & 0.132 & 14.5 & 8.7 & 16.157 & 1.96 & 264 & 2.97 & 40.00 & 39.63 & 8.6 \\
\hline SDSS J162806.20+084538.0 & 0.143 & 35.8 & 8.3 & 16.376 & 1.97 & 279 & 3.46 & 40.47 & 39.69 & 8.7 \\
\hline SDSS J162918.66+133824.0 & 0.118 & 72.0 & 15.6 & 16.093 & 1.96 & 249 & 3.31 & 40.59 & 39.78 & 8.5 \\
\hline SDSS J163043.14+163910.8 & 0.090 & 52.0 & 7.4 & 15.700 & 2.03 & 224 & 3.39 & 40.19 & 39.20 & 8.3 \\
\hline SDSS J164053.90+324728.4 & 0.136 & 50.0 & 5.4 & 16.458 & 1.97 & 226 & 3.19 & 40.56 & 39.45 & 8.3 \\
\hline SDSS J164548.45+393227.4 & 0.141 & 25.4 & 37.4 & 17.304 & 1.75 & 186 & 3.02 & 40.30 & 40.33 & 8.0 \\
\hline SDSS J164845.08+254119.5 & 0.115 & 102.6 & 8.8 & 16.201 & 2.02 & 228 & 3.13 & 40.72 & 39.50 & 8.4 \\
\hline SDSS J165304.98+400702.5 & 0.148 & 87.7 & 19.4 & 16.423 & 1.96 & 256 & 3.27 & 40.89 & 40.09 & 8.6 \\
\hline SDSS J165425.53+414121.2 & 0.147 & 17.2 & 6.8 & 17.283 & 1.91 & 201 & 3.04 & 40.17 & 39.62 & 8.1 \\
\hline SDSS J165448.44+261841.3 & 0.100 & 18.8 & 44.1 & 15.677 & 1.97 & 256 & 3.31 & 39.86 & 40.08 & 8.6 \\
\hline SDSS J165500.19+390847.9 & 0.139 & 54.8 & 17.5 & 15.887 & 2.05 & 358 & 3.42 & 40.63 & 39.99 & 9.1 \\
\hline SDSS J165744.77+215611.1 & 0.141 & 25.5 & 23.2 & 16.862 & 1.86 & 277 & 3.49 & 40.30 & 40.12 & 8.7 \\
\hline SDSS J170011.22+323514.7 & 0.102 & 185.0 & 9.1 & 15.734 & 1.87 & 239 & 3.26 & 40.86 & 39.41 & 8.4 \\
\hline SDSS J170115.59+240608.4 & 0.138 & 26.0 & 5.2 & 17.079 & 2.08 & 240 & 3.26 & 40.30 & 39.46 & 8.4 \\
\hline SDSS J170543.99+583001.2 & 0.114 & 26.9 & - & 16.436 & 1.97 & 183 & 3.20 & 40.13 & - & 8.0 \\
\hline SDSS J170602.20+201757.8 & 0.122 & 29.3 & 5.1 & 15.824 & 1.93 & 295 & 3.16 & 40.23 & 39.32 & 8.8 \\
\hline
\end{tabular}


Table B.1. continued.

\begin{tabular}{lcccccccccc}
\hline \hline & $z$ & NVSS & {$[\mathrm{O}$ III $]$} & $m_{r}$ & Dn & $\sigma_{*}$ & $C_{r}$ & $v L_{r}$ & $L_{[\mathrm{O} \text { III }]}$ & $M_{\mathrm{BH}}$ \\
\hline SDSS J171137.98+580330.2 & 0.147 & 42.0 & 18.6 & 16.354 & 2.11 & 312 & 3.32 & 40.56 & 40.06 & 8.9 \\
SDSS J171223.15+640157.1 & 0.080 & 150.0 & 10.8 & 15.848 & 2.02 & 235 & 3.03 & 40.55 & 39.26 & 8.4 \\
SDSS J171243.95+620245.0 & 0.122 & 47.2 & 19.4 & 16.243 & 1.86 & 274 & 3.35 & 40.43 & 39.90 & 8.7 \\
SDSS J173223.73+552452.8 & 0.062 & 54.8 & 42.6 & 15.093 & 2.09 & 247 & 3.24 & 39.88 & 39.62 & 8.5 \\
SDSS J212005.00-075350.1 & 0.139 & 48.2 & 22.4 & 16.844 & 2.02 & 263 & 3.21 & 40.57 & 40.09 & 8.6 \\
SDSS J214239.29-080423.8 & 0.128 & 13.9 & 8.9 & 16.904 & 1.95 & 231 & 3.19 & 39.95 & 39.61 & 8.4 \\
SDSS J223143.19-082431.7 & 0.083 & 766.0 & 61.1 & 14.149 & 2.01 & 301 & 2.86 & 41.29 & 40.04 & 8.8 \\
SDSS J234702.42-010300.9 & 0.133 & 34.3 & 5.8 & 17.507 & 1.83 & 202 & 2.98 & 40.38 & 39.46 & 8.1 \\
\hline
\end{tabular}

Table B.2. Properties of the sFRICAT sources.

\begin{tabular}{llrrlllllll}
\hline \hline & $\mathrm{z}$ & NVSS & [O III] & $m_{r}$ & Dn & $\sigma_{*}$ & $C_{r}$ & $v L_{r}$ & $\left.L_{[\mathrm{O}} \mathrm{III}\right]$ & $M_{\mathrm{BH}}$ \\
\hline SDSS J090100.09+103701.7 & 0.029 & 63.3 & 162.2 & 13.254 & 1.96 & 250 & 3.46 & 39.27 & 39.54 & 8.5 \\
SDSS J092122.11+545153.9 & 0.045 & 36.6 & 50.5 & 14.217 & 1.95 & 253 & 3.35 & 39.41 & 39.40 & 8.5 \\
SDSS J092151.48+332406.5 & 0.024 & 117.3 & 109.4 & 13.127 & 1.95 & 227 & 3.34 & 39.34 & 39.17 & 8.4 \\
SDSS J093957.34+164712.8 & 0.047 & 41.3 & 49.3 & 14.982 & 1.70 & 217 & 2.95 & 39.51 & 39.44 & 8.3 \\
SDSS J101623.01+601405.6 & 0.031 & 35.0 & 58.1 & 13.244 & 1.95 & 251 & 3.24 & 39.07 & 39.15 & 8.5 \\
SDSS J104740.48+385553.6 & 0.035 & 55.9 & 49.9 & 13.255 & 1.96 & 304 & 3.36 & 39.39 & 39.19 & 8.9 \\
SDSS J111125.21+265748.9 & 0.034 & 86.8 & 87.5 & 13.322 & 1.99 & 281 & 2.91 & 39.53 & 39.38 & 8.7 \\
SDSS J132451.44+362242.7 & 0.017 & 789.4 & 394.5 & 12.662 & 2.01 & 242 & 3.32 & 39.91 & 39.46 & 8.5 \\
SDSS J133242.54+071938.1 & 0.023 & 152.5 & 169.9 & 12.987 & 1.81 & 237 & 2.86 & 39.45 & 39.35 & 8.4 \\
SDSS J145222.83+170717.8 & 0.045 & 102.8 & 20.4 & 14.226 & 1.94 & 240 & 3.05 & 39.86 & 39.01 & 8.4 \\
SDSS J155603.90+242652.9 & 0.043 & 127.0 & 91.9 & 14.064 & 1.86 & 252 & 3.12 & 39.90 & 39.62 & 8.5 \\
SDSS J155749.61+161836.6 & 0.037 & 113.4 & 70.4 & 13.100 & 2.00 & 328 & 2.99 & 39.73 & 39.38 & 9.0 \\
SDSS J160332.08+171155.2 & 0.034 & 662.0 & 79.0 & 13.549 & 2.02 & 298 & 3.45 & 40.42 & 39.35 & 8.8 \\
SDSS J160722.95+135316.4 & 0.034 & 75.1 & 46.3 & 13.554 & 1.99 & 268 & 3.41 & 39.47 & 39.11 & 8.6 \\
\hline
\end{tabular}

Notes. Column description: (1) source name; (2) redshift; (3) NVSS $1.4 \mathrm{GHz}$ flux density [mJy]; (4) [O III] flux [in $10^{-17} \mathrm{erg} \mathrm{cm}^{-2} \mathrm{~s}^{-1} \mathrm{units}$; (5) SDSS DR7 $r$ band $A B$ magnitude; (6) concentration index $C_{r}$; (7) Dn(4000) index; (8) stellar velocity dispersion [km s ${ }^{-1}$; (9) logarithm of the radio luminosity [erg s${ }^{-1}$ ]; (10) logarithm of the [O III] line luminosity [erg s${ }^{-1}$ ]; (11) logarithm of the black hole mass [in solar units]. 